"Universitatea Alexandru Ioan Cuza", Iaşi

FaCUltatea DE Matematic $\breve{A}$

\title{
Advances in Computer Graphics
}

2014-2015 Master I

Applied Mathematics in Finance and Informatics 


\section{$0.1 \quad$ References}

1. Triangulations and Applications; Øyvind Hjelle, Morten Dæhlen; Springer 2006; Series Mathematics and Visualization

2. Computational Geometry: An Introduction; Franco P. Preparata, Michael Shamos; Texts and Monographs in Computer Science; Springer 1985

3. Algoritmi de triangulare;M.I.Munteanu, A.I.Nistor; Casa Editoriala Demiurg 2008

4. Pythagorean Hodograph Curves: Algebra and Geometry Inseparable;Rida T. Farouki; Springer 2008 


\subsection{Plan of the course}

- Triangulations:

$\rightarrow$ of a set of points

$\rightarrow$ Graham Scan method for a polygons

$\rightarrow$ Voronoi diagrams

$\rightarrow$ Delaunay triangulations

$\rightarrow$ Radial Sweep algorithm

$\rightarrow$ Aplications(art galery)

- Curves used in CAGD:

$\rightarrow$ Bézier, splines, B-splines, NURBS

$\rightarrow$ PH curves( planar and spatial PH curves) 


\section{Chapter 1}

\section{Triangulations}

A triangulation is made up of a collection of triangles satisfying certain restrictions. More precisely, we must enforce restrictions so that a collection of triangles, denoted by $\triangle$, becomes a subdivision of a domain into a collection of connected, non-overlapping triangles.

The triangles of a triangulation are formed by points given in a domain $\Omega$ of interest, which can be either be given or selected by some suitable procedure.

Usually, when constructing triangulation, we start with a given collection of points, say

$$
\mathcal{P}=\left\{p_{i}\right\}, i=1, \ldots, n
$$

and a domain $\Omega$ containing all the points in $\mathcal{P}$. We also assume that the boundary of $\Omega$ is one (or more) closed simple polygons. A simple polygon is a polygon that does not self-intersect. In many cases we prefer $\Omega$ to be the convex hull of the set of points.

\section{Notions:}

- point: geometric position in the plaine of a vertex in a triangulation;

- vertex or node of a triangulation denotes a topological element(which does not necessarily hold a geometric position)

- topology of a triangulation means relations between it's vertices, edges and triangles.

- points $p_{i}, p_{j}, p_{k}, \ldots \stackrel{\text { associate }}{\longrightarrow}$ vertices $v_{i}, v_{j}, v_{k}, \ldots$

- triangle $t_{i j k}$ or $t_{i, j, k}$ is spanned by three vertices $v_{i}, v_{j}, v_{k}$ such that $(i, j, k)$ is oriented namely the vertices are arranged counter clockwise around the triangle

- edges $e_{i j}$ or $e_{i, j}$ are not ordered

As we have already said, a collection of triangles $\triangle$ is called a triangulation if it satisfies certain requirements:

1. No triangle $t_{i j k}$ in $\triangle$ is degenerate, that is $p_{i}, p_{j}, p_{k}$ are not collinear.

2. The interiors of any two triangles in $\triangle$ do not intersect; that is

$$
\operatorname{Int}\left(t_{i j k}\right) \bigcap \operatorname{Int}\left(t_{\alpha \beta \gamma}\right)=\emptyset
$$


3. The boundaries of two triangles can only intersect at a common edge or a common vertex

4. The union of all triangles in a triangulation $\triangle$ is equal to the domain over which the triangulation is defined:

$$
\Omega=\bigcup t_{i j k}
$$

If these requirements are fulfilled, a triangulation is called VALID. However, we will work with the so called REGULAR triangulation and therefore supplementary conditions will be imposed:

5. The domain $\Omega$ must be connected

6. The triangulation $\triangle$ does not have "holes".

7. If $v_{i}$ is a vertex at the boundary $\partial \Omega$, then there exist exactly two boundary edges that have $v_{i}$ as a common vertex; as consequence, the $\sharp$ of boundary vertices and $\sharp$ boundary edges are the same.

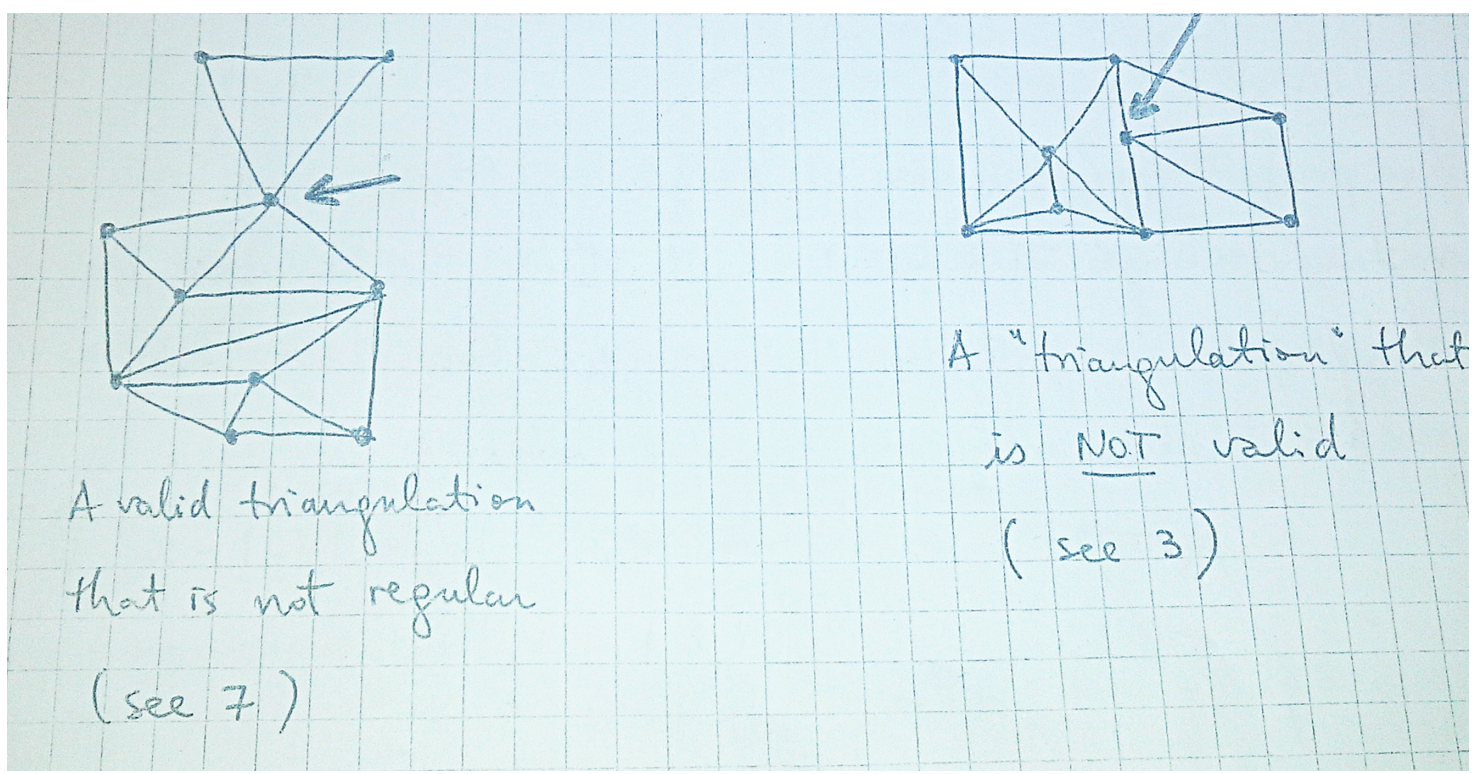




\subsection{Elementary properties of triangulations:}

Triangulations have interesting properties that can be deduced(many of them from the properties of plannar graphs theory.

For a triangulation $\triangle$ it is important to know "the size of $\triangle$ " useful when implementing algorithm for constructing it or for tranversing it.

We devive first some important relations between the number of vertices $|V|=\operatorname{card}(V)$, the number of edges $|E|=\operatorname{card}(E)$ and the number of triangles $|T|=\operatorname{card}(T)$, respectively. We will use subscripts I and B to denote interior elements and boundary elements, respectively, such as

- $V_{I}$ is the set of interior vertices

- $E_{B}$ is the set of boundary edges.

Obviously we have $V=V_{I} \bigcup V_{B}$.

Lemma 1. For a regular triangulation $\triangle$ we have

$$
\begin{gathered}
|T|=2\left|V_{I}\right|+\left|V_{B}\right|-2 \\
|E|=3\left|V_{I}\right|+2\left|V_{B}\right|-3 \\
\left|E_{I}\right|=3\left|V_{I}\right|+\left|V_{B}\right|-3
\end{gathered}
$$

Proof. By induction on the $\sharp$ of triangles: For $|T|=1$, i.e. for a triangle, we have

$$
\begin{aligned}
\left|V_{I}\right| & =0 ;\left|V_{B}\right|=3 \\
\left|E_{I}\right| & =0 ;\left|E_{B}\right|=3 .
\end{aligned}
$$

Let us prove the general case. Assume $|T| \geq 2$ and remove one boundary triangle t(from $\triangle$ ) such that the reduced triangulation $\hat{\triangle}$, is also regular. We have to consider two cases:

Case 1 t has only one boundary edge in $\triangle$

$$
\begin{gathered}
\left|\hat{V}_{I}\right|=\left|V_{I}\right|-1 \\
\left|\hat{V}_{B}\right|=\left|V_{B}\right|+1 \\
\left|\hat{E}_{I}\right|=\left|E_{I}\right|-2 \\
\left|\hat{E}_{I}\right|=\left|E_{I}\right|-1+2
\end{gathered}
$$

One interior point, that marked on the picture, was interior and became a boundary point.

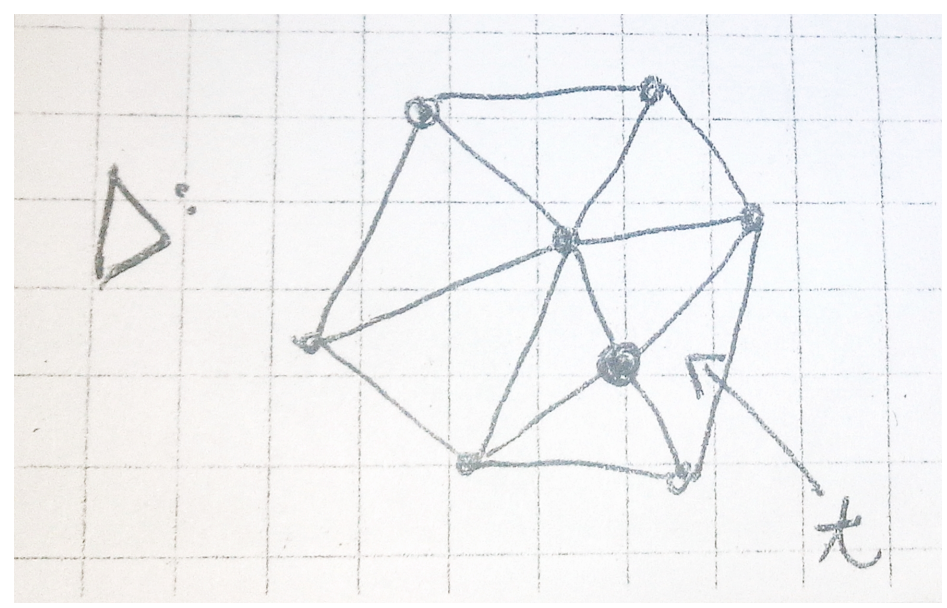


Case $2 \mathrm{t}$ has two boundary edges in $\triangle$ We have

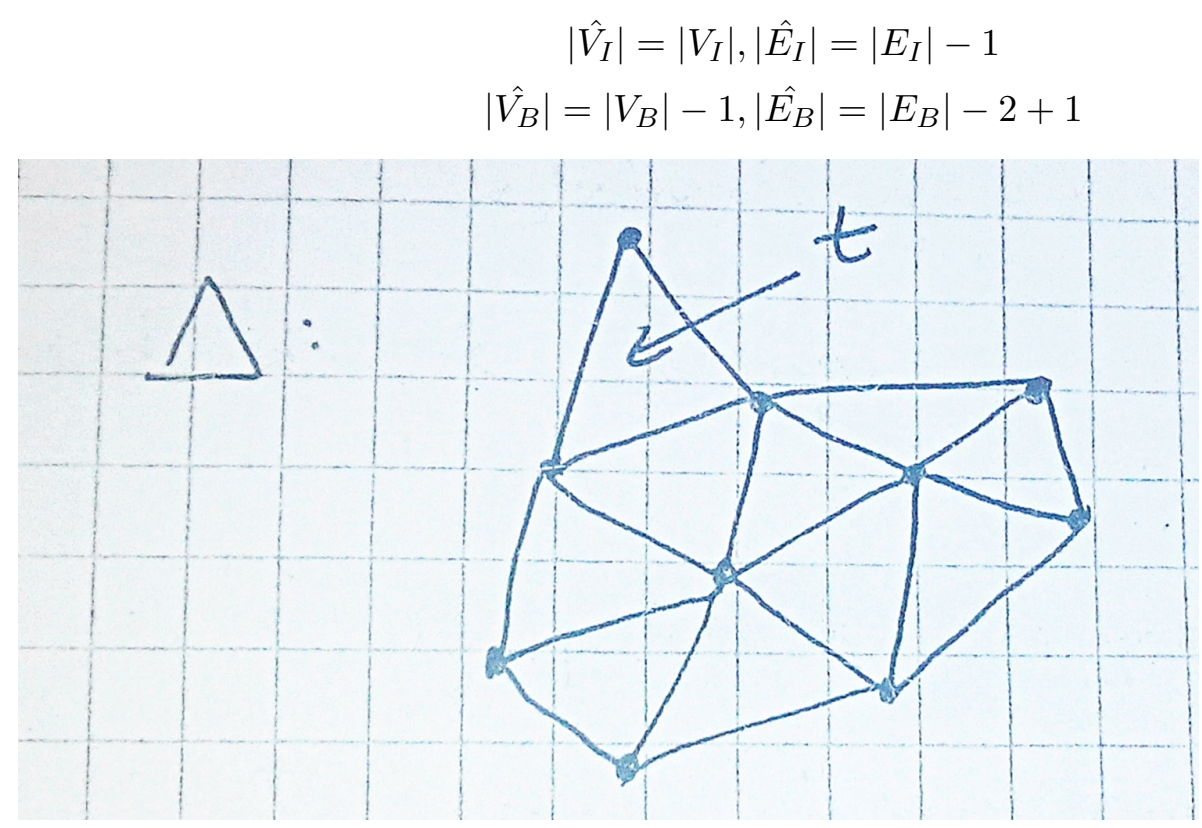

Using the corresponding relations between old and new values(together with the induction hypothesis) we got the conclusion.

Corollary 1. A special case of the Euler Polyhedron Formula, known also as the Euler-Poincaré formula:

$$
|T|=|E|-|V|+1
$$

Proof. Follows by combining previous relations.

Corollary 2. Adding an interior point in an existing triangulation:

- increases the number of triangles by 2

- increases the number of edges by 3

Lemma 2. (Gives lower and upper bounds on the number of triangles and edges) We have

$$
\begin{gathered}
|V|-2 \leq|T| \leq 2|V|-5 \\
2|V|-3 \leq|E| \leq 3|V|-6 .
\end{gathered}
$$

Proof. The key point is $\left|V_{I}\right| \geq 0$ while $\left|V_{B}\right| \geq 3$.

Another important property is related to the degree(sometimes called the valency) of a vertex in a triangulation $\triangle$. The degree of a vertex $v_{i}$ is denoted by $\operatorname{deg}\left(v_{i}\right)$ and is defined as the number of edges joining $v_{i}$ with another vertex in $\triangle$.

Lemma 3. In a triangulation $\triangle$ we have

$$
\sum_{i=1}^{|V|} \operatorname{deg}\left(v_{i}\right)=2|E| .
$$

Proof. Remark that every edge is incident with exactly 2 vertices. 


\subsection{Triangulation of a (finite) set of (planar) points}

The aim is to establish a triangulation of a set of points surrounded by a closed simple polygon. Given a set of points in the plane, without any closed polygon surrounding the point set, we can always construct such a polygon and this construction is, in general, not unique. One of such a polygon is the border of the convex hull; yet, the convex hull of a set of points can be determined (usually) by using triangulation methods.

Input data: a simple closed polygon and interior points.

Output data: $\triangle$, the triangulation of the set of points. Steps:

1. Contruct the triangulation of the (closed) polygon, without taking into account the interior points

2. Insert the interior points, one by one, keeping the triangulation updated as a regular triangulation for each insertion

3. Improve the triangulation by edge-swapping

1. Triangulation of a closed polygon:e.g. ear-cutting idea/method Graham Scan algorithm

- convex, concave vertex

- ear

- ear-cutting

2. Point insertion

$\rightarrow$ locate the triangle $t_{j}$ containing $p_{i}$ (such a triangle exists since the triangulations covers)

- the initial polygon, at the begining

- the domain, in what follows

$\rightarrow$ if $p_{i} \in \operatorname{Int}\left(t_{j}\right)$ replace $t_{j}$ with three new triangles:

$$
t_{j}=p_{a} p_{b} p_{c} \rightarrow \triangle p_{a} p_{i} p_{c} ; \triangle p_{a} p_{b} p_{i} ; \triangle p_{i} p_{b} p_{c}
$$

else if $p_{i} \in \operatorname{Int}\left(e_{k}\right)$, where $e_{k}$ is an edge of $t_{j}$, replace $t_{j}$ and the other triangle having $e_{k}$ with $t_{j}$ with four triangles.

Remark 1. The result depends on the order in which points are inserted, therefore the point insertion method does not give a unique triangulation.

Remark 2. (for practical use) In order to avoid some difficulties in working with numerical problems we have to take care when a point for insertion is very close to existing points and edges. Introduce a tolerance $\varepsilon$ and apply the following rules:

- If a point $p$ is such that dist $\left(p, p_{i}\right) \leq \varepsilon$, the point $p$ will not be inserted

- If a point $p$ is such that dist $\left(p, e_{k}\right) \leq \varepsilon$, insert the point as it belongs to the edge $e_{k}$.

3. The third step of the algorithm is used usually when we obtain long and skinny triangles. As we have already pointed out, the triangulation is not unique and it may considerably in shape. Hence we could improve the triangulation; e.g. decide that "a good triangulation" is one with as few small interior angles as possible. Since the position of the vertices are kept fixed, we must define new edges between vertices. One way to do this is to swap edges which are diagonals in strictly convex(all its four interior angles are less than $180 \circ$ quadrilaterals. 
The algorithm:

1. Locate a diagonal of a strictly convex quadrilateral

2. Swap the diagonal if the smallest of the six interior angles within the quadrilateral increases

3. If all strictly convex quadrilaterals have been checked and no swaps have been performed:STOP, else go to STEP 1.

\subsection{Constrains}

Predefined edges in triangulations are frequently used(for representing rivers, roads) and are commonly referred as constrained edges. A triangulation with predefined edges is called constrained triangulation. Construct a triangulation without taking into the constraints, represents the initial regular triangulation.

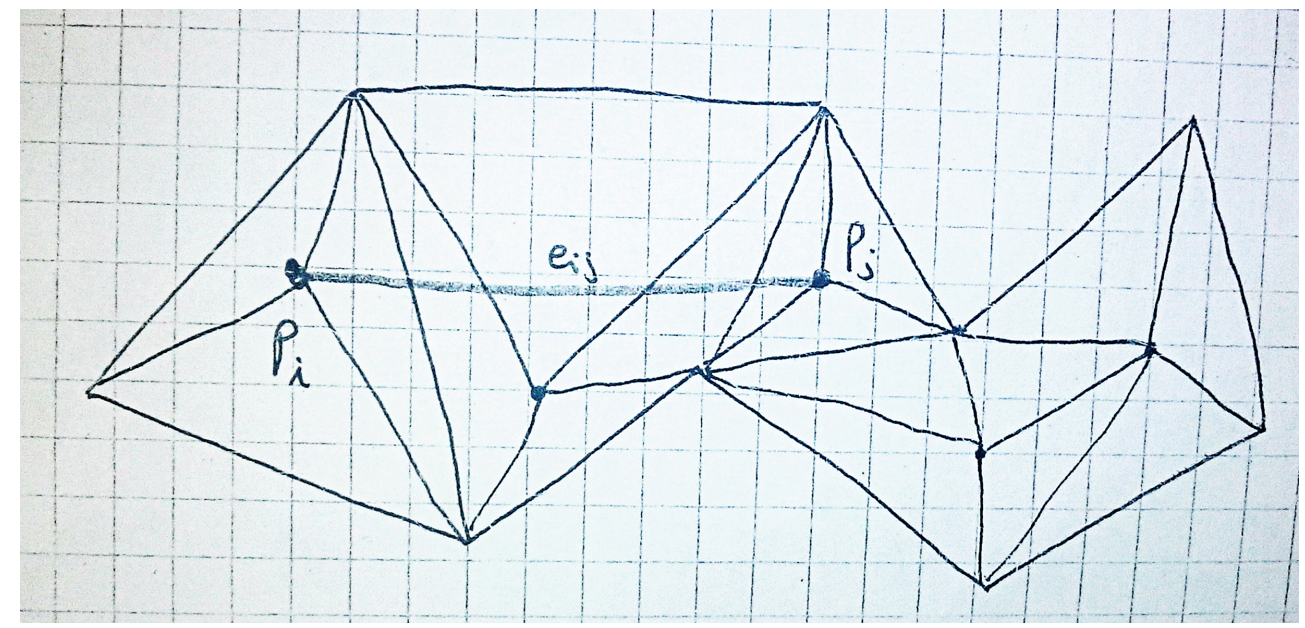

We want to insert the edge $e_{i j}$ in the triangulation such that the resulting triangulation is valid and regular. We start to remove the edges that are intersected by $e_{i j}$, or more precisely, all triangles that are intersected by $e_{i j}$ will be removed from the initial triangulation. The removed triangles determine a region, usually, called the influence region of $e_{i j}$.

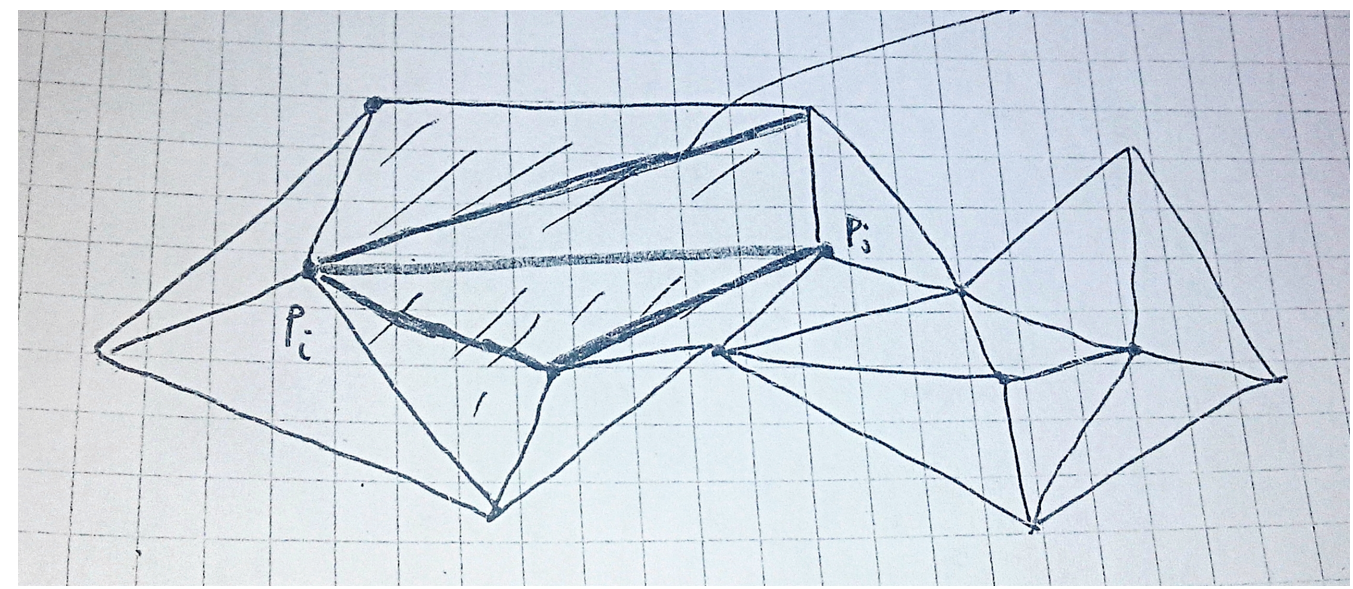

What do we have next? One has to triangulate the influence region such that the constrained edge $e_{i j}$ is included in the set of edges of the triangulation. The influence region is made by more 
regions which are simple closed polygons(with their interiors) and we have to divide all these regions into triangles.

\subsection{Voronoi Diagrams}

In computational geometry one finds the so called The Post Office Problem (assign to residence the nearest post office) or NNS-nearest neighbour search or proximity search (given a set $S$ of points - in a certain space $X$ - and a fixed point $q \in X$ find the closest point in $S$ to $q$ ).

\section{Problem}

Suppose that the advisory board of a supermarket chair plans to open a new branch at a certain location. To predict whether the new branch will be profitable, one has to estimate the numbers of customers it will attract. For this, we have to model the behavior of the potential customers: how people decide where to do their shopping?

In a more abstract setting we have a set of central places called sites that provide certain goods or services, and we want to know, for each site, where the people live, who obtain their goods or services from that site. E.g. post office: customers want to post their letters.

To study this problem we have to make some simplifying assumptions:

1) the price of particular good or service is the same at every site;

2) the cost of acquiring the good or service is equal to the price plus the cost of the transportation to the site;

3) the cost of the transportation to the site equals the Euclidean distance to the site times a fixed price per unit distance;

4) customers try to minimize the cost of acquiring the good or service.

Usually, these assumptions are not completely satisfied:

- goods may be cheaper at some sites than at others

- inside a city the transportation cost from one point to another is (probably) not linear in the Euclidean distance between the points

Our interest is in the geometric interpretation of the model. The assumptions we made induce a subdivision of the total area under consideration into regions such that people who live in the same region all go to the same site. This means that people simply get their goods at the nearest site, which means that the area corresponding to a given site consists of all those points for which that site is closer than any other site.

The model where every point is assigned to the nearest site is called the Voronoi assignement model. The subdivision induced by this model is called the Voronoi diagram of the set of sites.

From the Voronoi diagram we can derive several information about the sites and their relations. For example, if the regions corresponding to two sites have a common boundary, then these two sites are in competition for customers living in the boundary region. 


\subsubsection{Definition and basic properties}

Consider a set of distinct points $P=\left\{p_{1}, p_{2}, \ldots, p_{n}\right\}$ called sites; $d\left(p_{i}, p_{j}\right)$ denotes the Euclidean distance between $p_{i}$ and $p_{j}$.

To each point $p_{i} \in P$, we associate a region in the plane:

$V\left(p_{i}\right)=\left\{p: d\left(p, p_{i}\right) \leq d\left(p, p_{j}\right) \forall j=1, \ldots, n\right\}$ called the Voronoi region of $p_{i}$. That is, $V\left(p_{i}\right)$ consisits of all points in the plane that are closer to $p_{i}$ than to any other point in $P$.

Let $H(p, q)$ be the half plane defined by the perpendicular bisector of the line $[p, q]$ which contains $p$; the other half plane is $H(q, p)$.

Note that, if $r \in H(p, q)$ then $d(p, r)<\operatorname{dist}(q, r)$. The converse is also true.

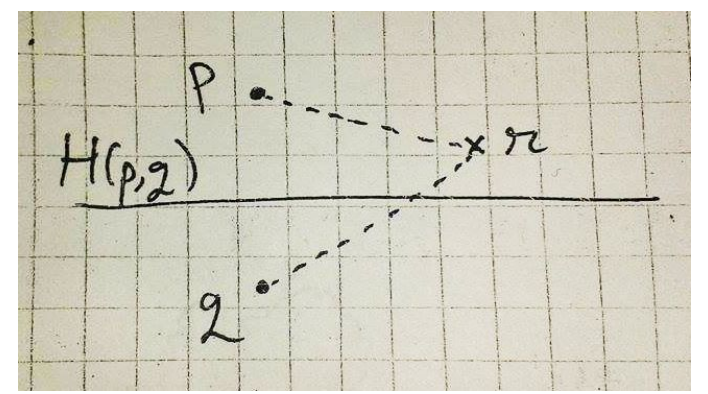

Therefore, $V\left(p_{i}\right)=\bigcap_{j \neq i} H\left(p_{i}, p_{j}\right)$ i.e. a intersection of n- 1 half planes which contain (all) the point $p_{i}$.

Consequences:

1. Each Voronoi cell (region) is a convex set

2. There exist bounded regions, as well as unbounded regions.

If we do in such way, namely if we apply this construcion to all points in $P$, we obtain a unique set of non-intersecting (this refers to the interior of Voronoi cells) Voronoi regions $V\left(p_{i}\right), i=1, \ldots, n$

Input data: $P$

Output data: $\operatorname{Vor}(P)=\bigcup_{i=1}^{n} V\left(p_{i}\right) \rightarrow$ the Voronoi diagram.

Remark 3. The boundaries of the Voronoi regions are line segments, half-lines or lines.

Theorem 1.1. Let $P$ be a set of $n$ sites in the Euclidean plane.

1) if all sites are collinear, then $\operatorname{Vor}(P)$ consists in $n$-1 straight lines

2) otherwise, the Voronoi edges are half-lines and segments (and we get a connected graph).

Proof 1. 1) Obvious

2) Suppose, by contradiction, that one Voronoi edge is a straight line and denote it by e. It represents the common boundary of two cells $V\left(p_{i}\right)$ and $V\left(p_{j}\right)$. 


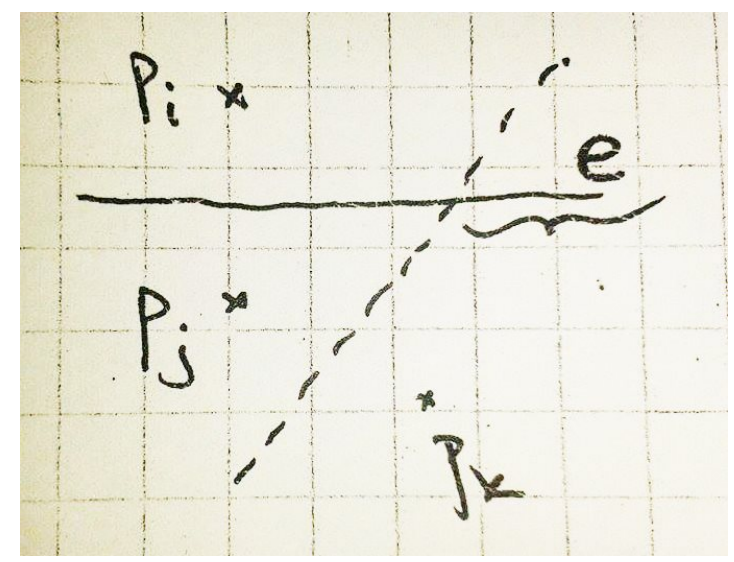

As not all sites are collinear, let $p_{k}$ such that $p_{i}, p_{j}$ and $p_{k}$ are not collinear. It follows that the perpendicular bisector of $p_{j} p_{k}$ is not parallel to e; thus they intersect. Therefore, a portion of e, lying in the half plane $H\left(p_{k}, p_{j}\right)$ cannot belong to the boundary of $V\left(p_{j}\right)$ since all points are closer to $p_{k}$ than to $p_{j}$.

\section{Exercise}

Give an example of a Voronoi diagram, where $n \geq 4$ ( with at least four non parallel sites ) such that all cells are undounded.

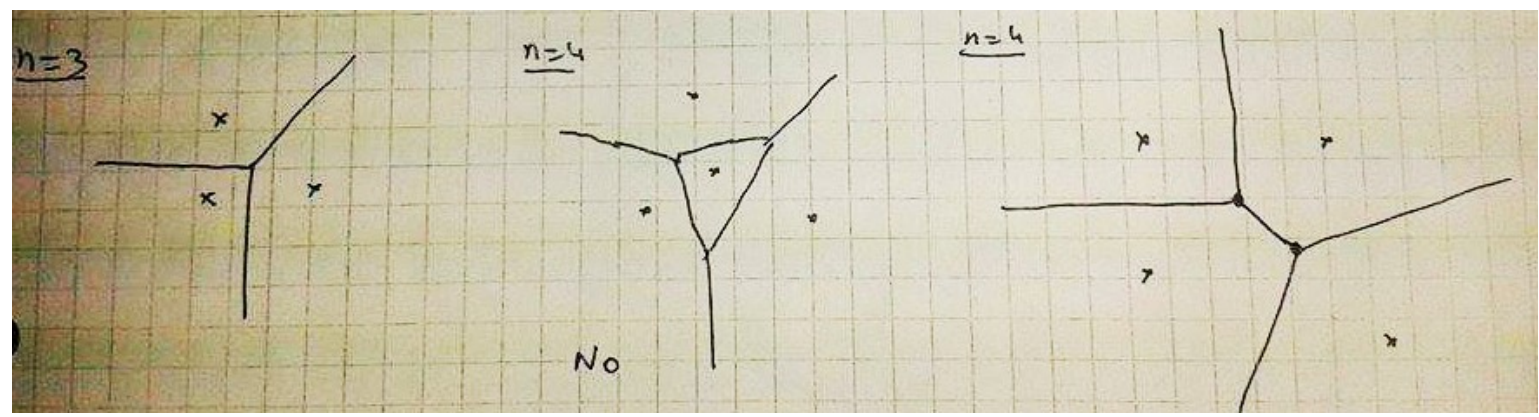




\subsubsection{The complexity of a Voronoi diagram}

Theorem 1.2. Let $n \geq 3$ be the number of sites (as before) in the plane. Then, the number of vertices $N$ of the Voronoi diagram is at most $2 n-5$, and the number of Voronoi edges at most $3 n-6$.

Proof. The case when all sites are collinear follows immediately:

$$
N=0, N_{E}=n-1
$$

Suppose that all three sites are not collinear.

Recall that for a planar connected graph we have the Euler formula:

$$
N_{V}-N_{E}+N_{F}=2
$$

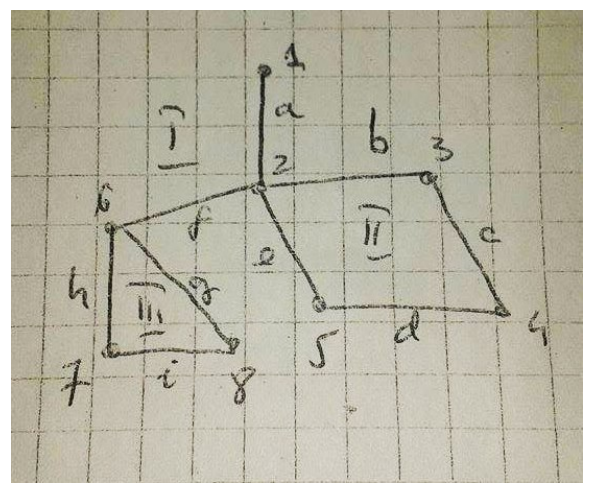

$\left(N_{V}=\#\right.$ nodes $/$ vertices $; N_{E}=\#$ edges $; N_{F}=\#$ faces $)$

Yet, in the case of a Voronoi diagram we cannot use this formula directly, because the edges of $\operatorname{Vor}(p)$ can be half-lines and hence it is not a proper graph.

Nevertheless, if we add an imaginary point called infinity $(\infty)$ we are able to use graph theory.

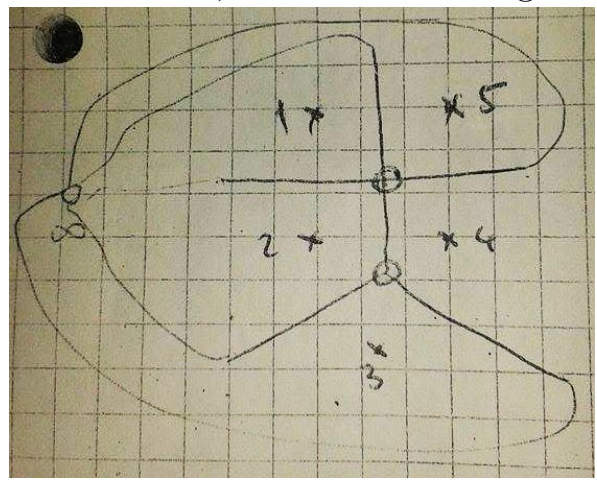

In this situation,

$$
\begin{gathered}
N_{V}=N+1, \\
N_{F}=n,
\end{gathered}
$$

hence, we have

$$
\underline{N+1-N_{E}+n=2} \Rightarrow N_{E}=N+n-1 .
$$


On the other hand, to each Voronoi edge corresponds exactly two nodes of this graph (including $\infty$ ), hence $\sum \operatorname{deg}\left(v_{i}\right)=2 N_{E}$.

But each vertex of the diagram is situated to equal distance at least three points ( sites ), hence

$$
\begin{gathered}
\operatorname{deg}\left(v_{i}\right) \geq 3 \Rightarrow \sum \operatorname{deg}\left(v_{i}\right) \geq 3(N+1) \\
2(N+n-1) \geq 3(N+1) \Rightarrow N \leq 2 n-5 \\
N_{E}=N+n-1 \leq 3 n-6 .
\end{gathered}
$$

Question:When the equality case holds?

$\rightarrow$ Each node is situated at the same distance with regard to exactly three locations ( these three points always exist since any three points are concyclic), but there do not exist four points located on the same circle. 


\section{Some examples:}

1) $N=1 ; N_{E}=4 ; n=4 ; 2 n-5=3 ; 3 n-6=6$

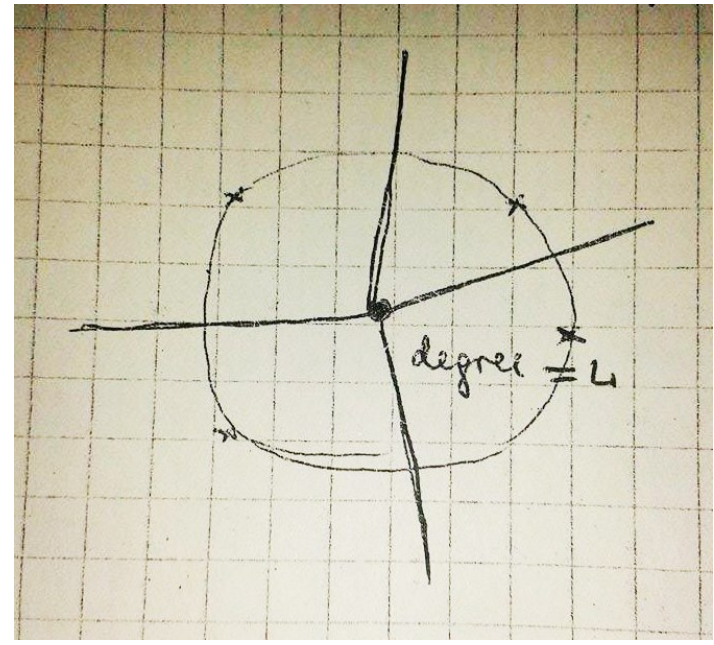

2) $N=2 ; N_{E}=5 ; n=4 ; 2 n-5=3 ; 3 n-6=6$

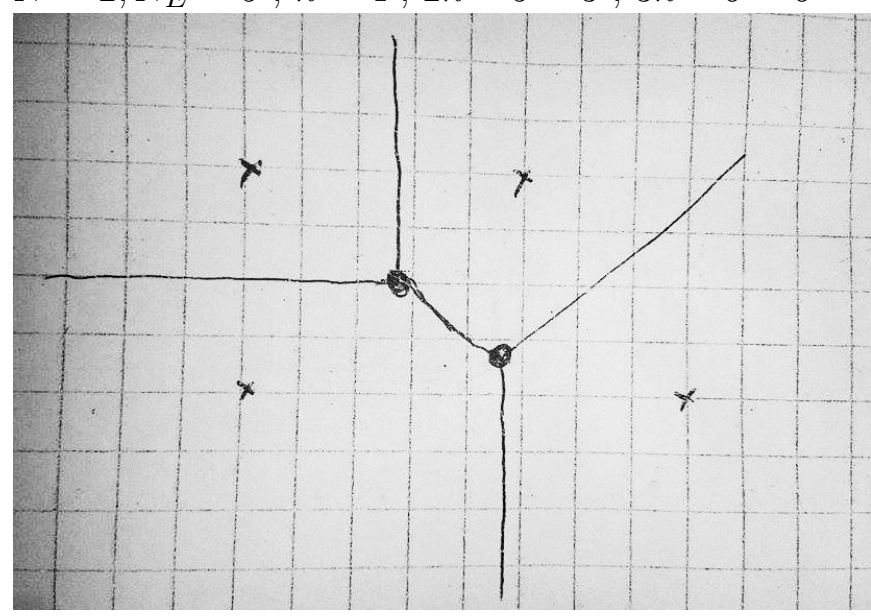

Question:Why one does not have equality case?

!Answer:The infinity point should have also the degree 3 , that is the number of half-lines must be 3 .

3) $N=3 ; N_{E}=6 ; n=4 ; 2 n-5=3 ; 3 n-6=6(\infty$ has also degree $=3)$

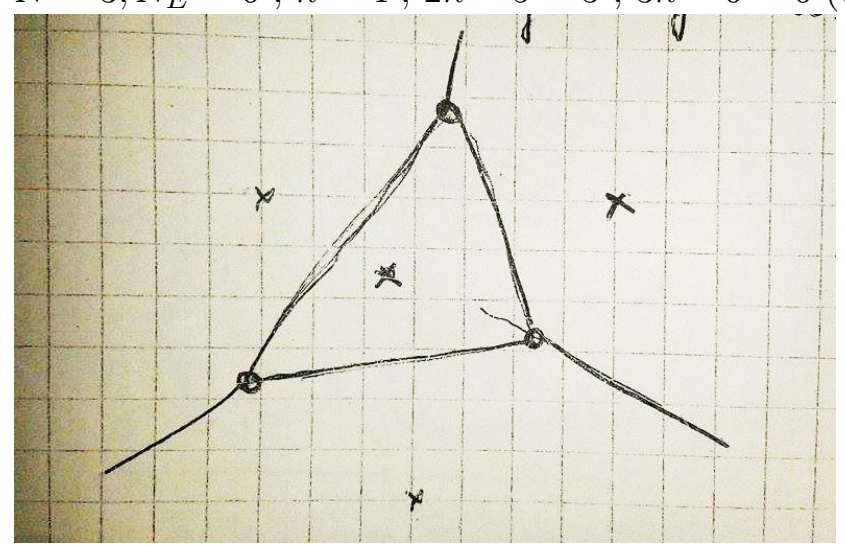




\subsubsection{Characterizations for edges and nodes}

From now on we suppose that there are no 4 concyclic points.

As immediate consequence, every node has degree 3 ( prove this fact!).

Definition 1.1. For an arbitrary point $q$ (in the plane) define the longest empty circle with regard to $P$ and demoted by $C_{P}(q)$ as the circle centered in $q$, of maximum radius and containing no sites of $P$ inside.

Theorem 1.3. 1) A point $q$ is a node of $\operatorname{Vor}(P)$ if and only if the boundary of the largest empty set $C_{P}(q)$ contains at least 3 sites (in fact precisely 3 ).

2) The orthogonal bisector of $\left[p_{i} p_{j}\right]$ defines on edge of the Voronoi diagram Vor $(P)$ ( namely contributes to the diagram ) if and only if $\exists$ q on it such that $p_{i}$ and $p_{j}$ belong to the boundary of the largest empty circle $C_{P}(q)$ and no other site belongs to it.

\section{Proof. (sketch)}

1) Suppose that the boundary circle contains 3 or more sites $p_{i}, p_{j}, p_{k}$. It follows that $\operatorname{dist}\left(q, p_{i}\right)=\operatorname{dist}\left(q, p_{j}\right)=\operatorname{dist}\left(q, p_{k}\right)$.

Moreover, being the largest empty circle, no other site $p_{l}$ belongs to the interior of the disc, therefore, there is no other site closer to $q$.

Hence, $q$ belongs to the boundary of each cell $V\left(p_{i}\right), V\left(p_{j}\right)$ or $V\left(p_{k}\right)$.

Conversely, each node $q$ of the Voronoi diagram $\operatorname{Vor}(P)$ has degree 3 , that is it is an end for 3 edges.

Therefore there exist 3 cells $V\left(p_{i}\right), V\left(p_{j}\right)$ and $V\left(p_{k}\right)$ incident at $q$ and there is no other location (site) closer to q. Subsequently, $C_{P}(q)$ contains exactly these three sites.

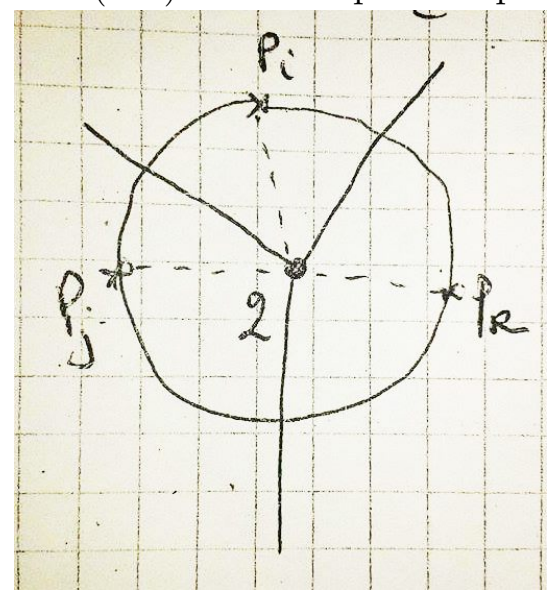

2) Similar discussions. 


\subsubsection{How can we construct the Voronoi diagram?}

Of course we can use direct approach to construct the Voronoi diagram $\operatorname{Vor}(P)$, construct individually each cell. In such a way we obtain an algorithm whose complexity is $O\left(n^{2} \log n\right)$.

We will present an algorithm, know as the Fortunes algorithm or sweep line algorithm whose complexity is $O(n \log n)$. Moreover, it can be proved that one cannot obtain a better complexity, so, this algorithm is optimal.

The idea of this algorithm is the following: move a line (sweep line) horizontally, from top to bottom over the plane. The most important thing is that the information does not change essentially in general, but only at certain special points called event points.

How do we proceed? Move a horizontal sweep line $l$ and compute its intersection with the Voronoi diagram. Unfortunately this is not so easy, because the part of $\operatorname{Vor}(P)$ above $l$ depends not only on the sites that lie above $l$, but also on sites below $l$. More precisely, when the sweep line cuts the cell $V\left(p_{i}\right)$, the site $p_{i}$ lies below $l$, so, in order to have information about intersections of $l$ with the boundary of the Voronoi cell $V\left(p_{i}\right)$ we do not have all information about that cell. Therefore, we are forced to apply this strategy in a slightly different way: instead maintaining intersections of the Voronoi diagram with the sweep line maintain information about that point of the Voronoi diagram of the sites above $l$ that cannot be changed by the sites below $l$.

Denote by $l^{+}$the closed half plane situated above $l$

Question: Which is the part of the Voronoi diagram ( situated in $l^{+}$) that cannot be changed anymore?

In other words, for which points $q \in l^{+}$do we know for sure what the nearest site is?

Remark 4. Obviously, the distance from $q \in l^{+}$to any site situated below $l$ is strictly greater than the distance from $q$ to $l$.

Therefore, the closest site of $q$ cannot be located below $l$ if $q$ is at least as near to some site $p_{i} \in l^{+}$ as $q$ is to $l$.

We obtain a problem to solve:

Problem Let $p_{i} \in l^{+}$

Find the locus of points that one closer to $p_{i}$ than the sweep line $l$.

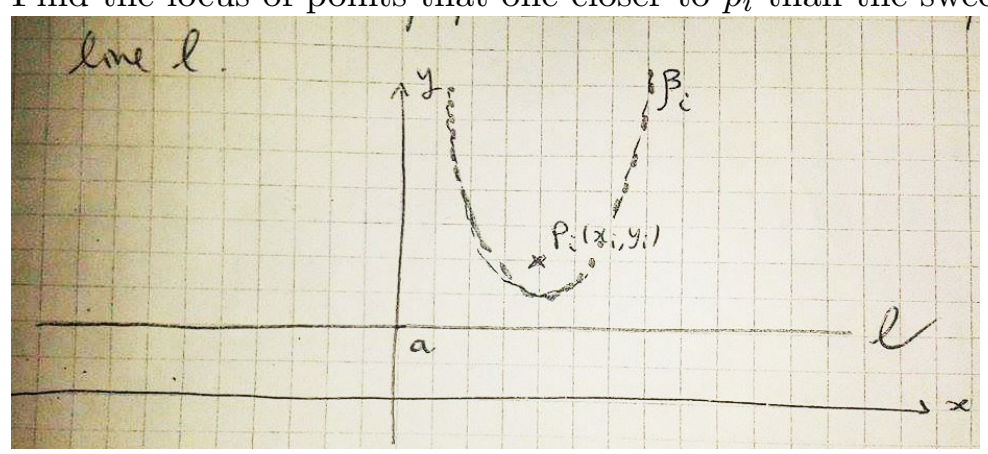

Find $q=(x, y)$ such that $\operatorname{dist}(q, l)=\operatorname{dist}\left(q, p_{i}\right)$. 
We have

$$
\begin{gathered}
\left(x-x_{i}\right)^{2}+\left(y-y_{i}\right)^{2}=|y-a|^{2} \\
y=\frac{\left(x-x_{i}\right)^{2}+y_{i}^{2}-a^{2}}{2\left(y_{i}-a\right)}
\end{gathered}
$$

This is the equation of a parabola, with $V_{i}\left(x_{i}, \frac{y_{i}+a}{2}\right)$ which we denote by $\beta_{i}$.

Notice that for $a=y_{i}\left(p_{i} \in l\right)$, the previous parabola degenerates into two half lines (which coincide): $x=x_{i}$ (double line).

Therefore, the set of all points which are close to $p_{i}$ than the sweep line $l$ is the set of all points located above the parabola $\beta_{i}$.

Hence, the set of points which are closer to sites located above $l$ than the sweep line $l$ is bounded by the so called the beach line, that is a union of arcs of parabolas.

Homework: Write the equation of the locus of points situated on both parabolas $\beta_{i}$ and $\beta_{j}$ when the sweep line moves from top to bottom.

Definition 1.2. Points where two arcs of parabola meet (from the beach line) is called breakpoint.

Remark 5. This points draw the Voronoi diagram, as they lie on an edge of the Voronoi diagram.

\section{How can we get the beach line?}

For every $x$, consider the smallest $y$ among all parabolas that is, the closest point to the sweep line. In such a way, we are no more interested in the intersection of the sweep line with the Voronoi diagram, but in the movement of the beach line when sweep line is moving.

\section{When the beach line modifies?}

I. when a new arc of parabola appears

II. when an existing arc of parabola shrinks to a point and disappears

\section{How the beach line modifies?}

I. When a new arc appears, that is when the sweep line meets a new site $p_{j}$, a new parabola $\beta_{j}$ appears: initially it is a degenerate parabola namely a double vertical segment, which enlarges while the sweep line goes ahead.

PROVE THIS FACT! 


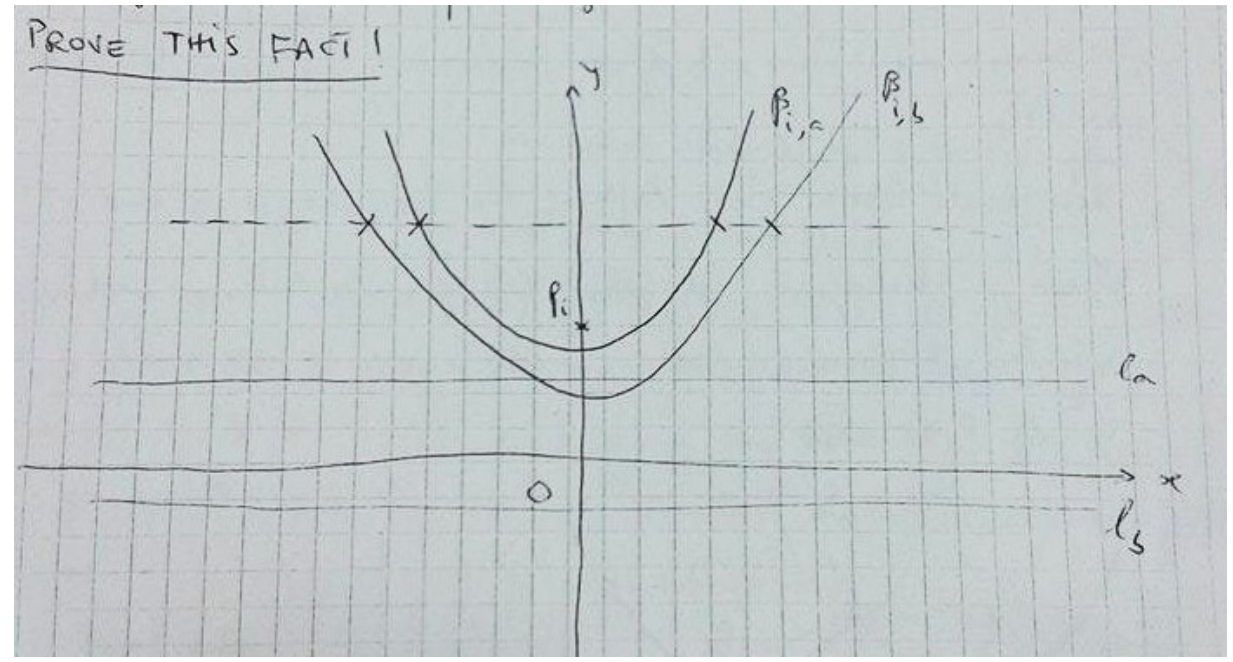

In such a way, the new parabola appears as shown in the next picture:

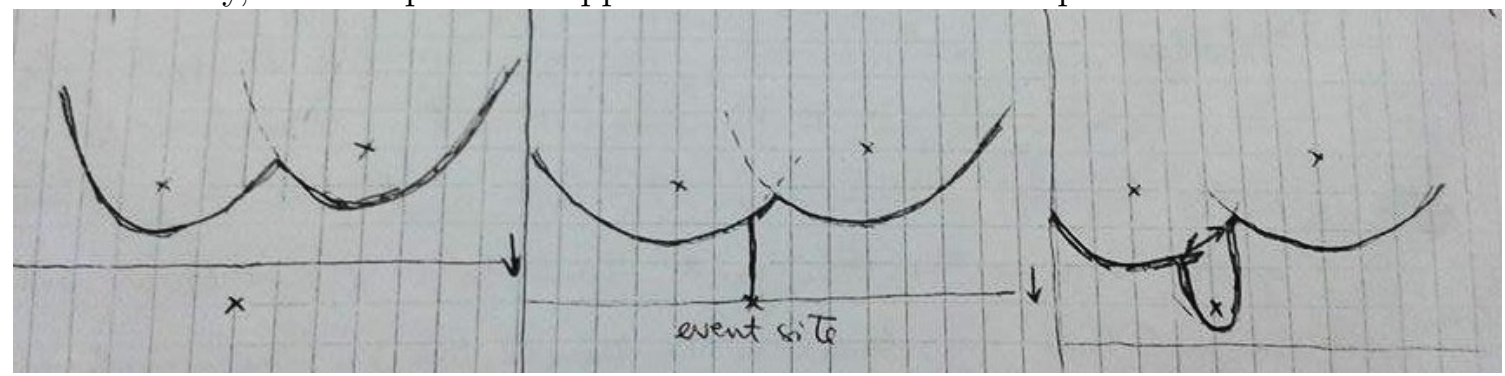

Remark 6. 1) A parabola may contribute more times in constructing the beach line ( namely with several pieces)

2) Lemma: The only way when a new arc may appear is through an event site.

3) As consequence, the beach line can have at most 2n-1 arcs. item [4)] Every new site (encountered by the sweep line) yields a splitting in two parts of an exciting arc (two breakpoints appear, initially they coincide).

II. What happens when an arc (on the beach line) shrinks to a point and disappears?

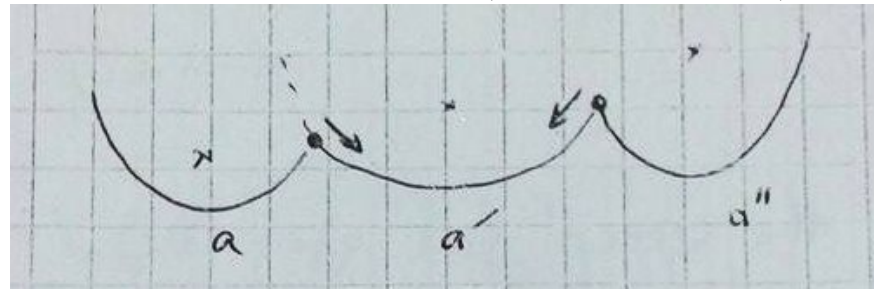

The two arc $a$ and $a^{\prime \prime}$ force $a^{\prime}$ to shrink; moreover they cannot belong to the same parabola. There exist three (distinct) sites defining the three arcs $a, a^{\prime}$ and $a^{\prime \prime}$.

When $a^{\prime}$ disappears, it follows that $a$ and $a^{\prime \prime}$ have a common point q situated at the same distance with respect to the sweep line $l$ and to all three sites. 


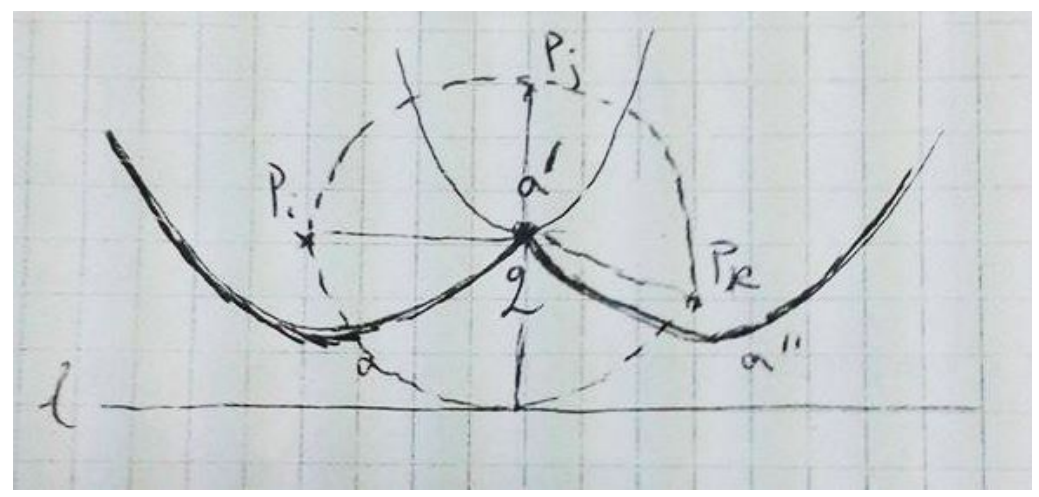

Therefore, there exists a circle passing through $p_{i}, p_{j}$ and $p_{k}$ which is also tangent to the sweep line $l$. Moreover, this circle does not contain any other site in its interior, otherwise $q$ will be closer to this point than to $l$ ( and this contradicts the fact that $q$ belongs to the beach line ). One concludes that this is the largest empty circle $C_{p}(q)$ and it contains three sites on the boundary. Hence $q$ is a vertex of the Voronoi diagram.

Remark 7. $a \bigcap a^{\prime}$ defines an edge of the Voronoi diagram.

$a^{\prime} \cap a^{\prime \prime}$ defines an edge of the Voronoi diagram.

They melt in a vortex $q$.

Definition 1.3. We call this event: circle event when the sweep line is tangent to a circle defined by 3 sites (at its bottom point). 


\subsection{Delaunay triangulation}

Definition 1.4. $\quad *$ is a regular triangulation (that is all axioms are satisfied)

* each edge is legal.

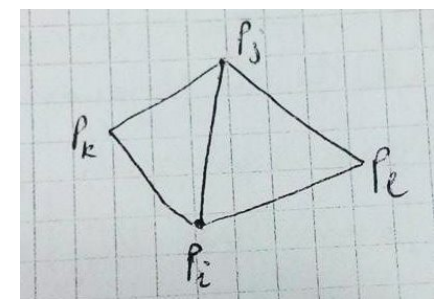

Let us explain this concept:

Let $\left[p_{i} p_{j}\right]$ be an edge common to both triangles $t_{i l j}$ and $t_{i j k}$.

Consider $C$ the circle passing through $p_{i}, p_{j}$ and $p_{k}$.

We say that the edge $\left[p_{i} p_{j}\right]$ is illegal (and it will not be allowed ) if and only if point $p_{l}$ is situated in the interior of $C$.

Remark 8. 1) If the 4 points $p_{i}, p_{k}, p_{j}, p_{l}$ are the vertices of a convex quadrilater and they are NOT situated on the same circle, then one and only one of the edges (diagonals) $\left[p_{i} p_{j}\right]$ and $\left[p_{l} p_{k}\right]$ is an illegal edge.

2) The criterion used in giving the definition is symmetric in $p_{k}$ and $p_{l}$. Let us give more details: as the four points are not concyclic we have either.
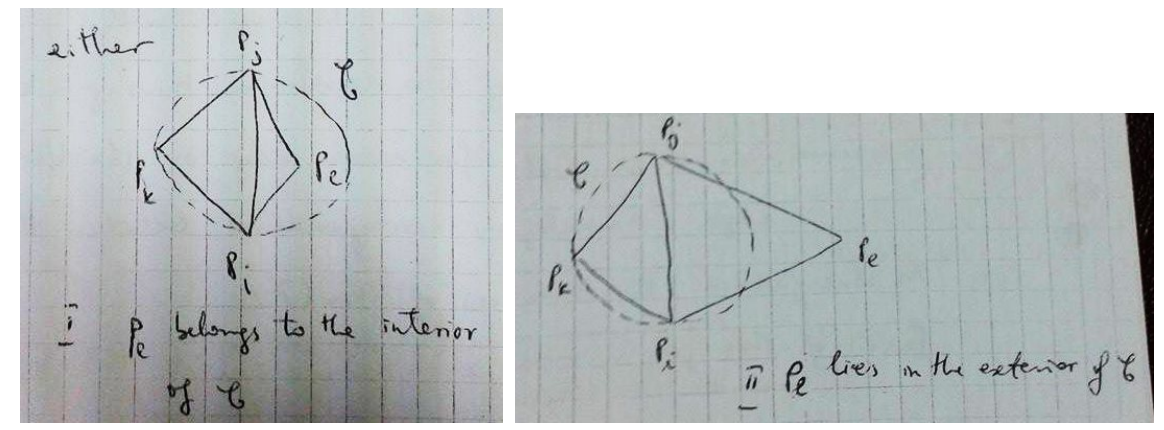

In case 1) $\left[p_{i} p_{j}\right]$ is an illegal edge.

It is an elementary exercise to prove that this fact is equivalent to: $m\left(\measuredangle p_{k}\right)+m\left(\measuredangle p_{l}\right)>\pi$ From this inequality one has :

- $m\left(\measuredangle p_{i}\right)+m\left(\measuredangle p_{j}\right)<\pi$, meaning that the two diagonals $\left[p_{i} p_{j}\right]$ and $\left[p_{l} p_{k}\right]$ have a different behavior.

- the symmetry in $p_{k}$ and $p_{l}$.

Definition 1.5. We call a legal triangulation a general triangulation which contains no illegal edge.

Let us now go back to the Voronoi diagram of a set of $n$ planar points: it represents a subdivision of the plane, into n regions, one for each site, such that $V\left(p_{i}\right)$ contains all points situated closer to $p_{i}$ than to any other $p_{j}$. 


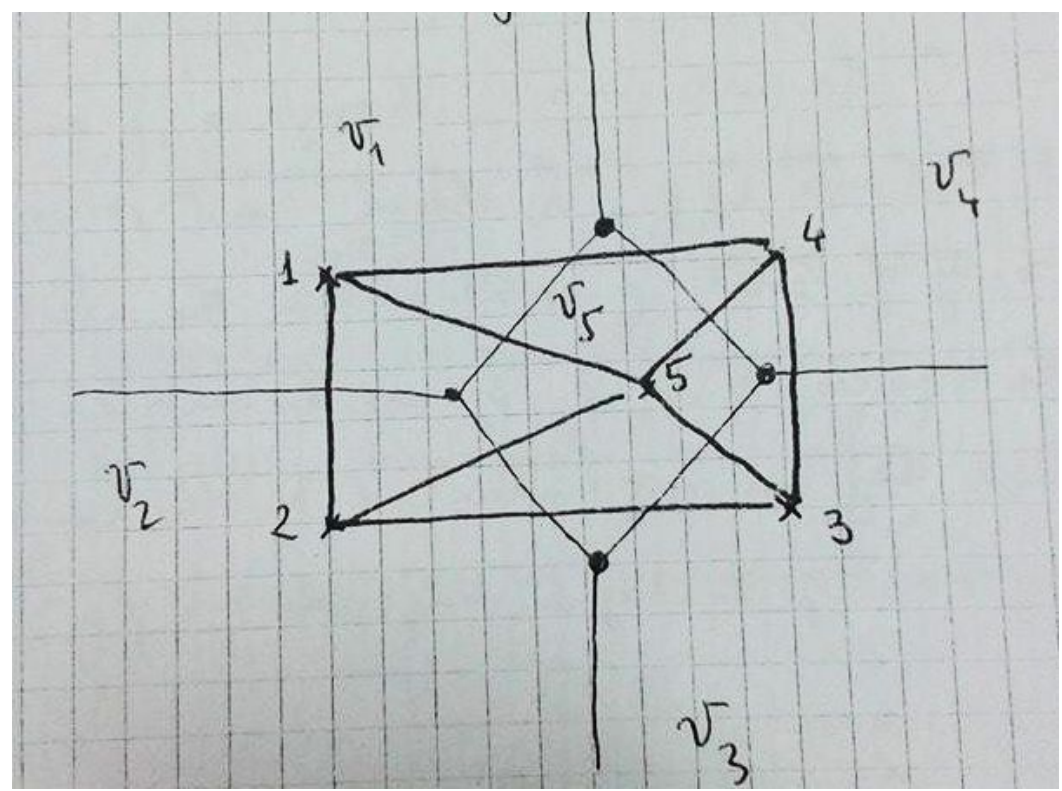

We construct now the "dual graph" of the Voronoi diagram:

* one vertex (node) for each Voronoi cell

* one edge between every two location having a common edge, namely an edge for each edge in the $\operatorname{Vor}(P)$.

As consequence, there is a one-to-one correspondence between the bondery faces of this graph and the vertices of $\operatorname{Vor}(P)$.

If one consider as nodes the sites $p_{i}$ and as edges the line segments joining the corresponding sites, one obtains the so called DELAUNAY GRAPH: DG(P).

Theorem 1.4. (without proof) The Delaunay graph $D G(P)$ is a planar graph.

$\underline{\text { Sketch }}$

See the analogy between the largest empty circle (used in the construction of the Voronoi diagram) and the definition of an illegal edge.

Definition 1.6. The Delaunay triangulation of a set of planar points $P$ is given as the dual graph of the corresponding Voronoi diagram, where the vertices are sites in $P$ and edges are line segments joining the corresponding sites.

Proposition 1.1. (without proof) The two triangulations we have obtained:

* as the dual graph of the Voronoi diagram

* as the optimal triangulation with respect to the MaxMin criterion

coincide.

$\underline{\text { Conclusion regarding duality }}$ 
* A Voronoi node is equidistant to exactly 3 neighbors from $P$. These 3 neighbors define a Delaunay triangle. In such a way, the Voronoi node is the center of the circumcircle of the Delaunay triangle. Therefore, to each Voronoi node one associates a Delaunay triangle.

* In particular situations, when we do have 4 concyclic points, the Delaunay triangulations is not unique.

* Hence:

a) a Voronoi node $\Rightarrow$ Delaunay triangle

b) a Voronoi edge $\Rightarrow$ Dalaunay edge

c) a Voronoi polygon (cell) $\Rightarrow$ Delaunay node (site)

d) The circumcirlce criterion for the Delaunay triangulation:

A Delaunay triangulation of a set of points $P$ in a triangulation $\Delta$ for which the interior of the circumcircle of every triangle in $\Delta$ contains no points in $\mathrm{P}$.

$\underline{\text { Homework }}$

How a Delaunay triangulation changes, when a new site is inserted? 


\section{Chapter 2}

\section{PH curve}

\subsection{Pythagorean hodograph curves}

For the beginning we will consider only planar curves.

Suppose we have a curve parametrized as $r(t)=(x(t), y(t)), t \in I$

Definition 2.1. The hodograph of $r$ is the locus of $r^{\prime}(t)=\left(x^{\prime}(t), y^{\prime}(t)\right), t \in I$ (we consider that $r$ is $C^{\infty}$ )

Without loss of the generality one can suppose that $r$ is a polygon curve ( Weirstrass theorem ) .

Definition 2.2. We say that $r$ has Pythagorean hodograph (or $r$ is a PH curve) if there exist a polynomial $\sigma$ such that $x^{\prime}(t)^{2}+y^{\prime}(t)^{2}=\sigma(t)^{2}$ for all $t \in I$.

Remark 9. Recall that $l($ curve $)=\int_{a}^{b}\left|r^{\prime}(t)\right| d t\left(r^{\prime}(t) \rightarrow \sqrt{x^{\prime 2}+y^{\prime 2}}=|\sigma|\right)$.

So, the arc length is expressed as a polynomial in $t$.

Pythagoras Theorem $a^{2}+b^{2}=c^{2}$ (in $\mathbb{R}$ says nothing interesting; in $\mathbb{Z} \rightarrow$ Pythagorean triples). When we are working with functions instead numbers? For continuous functions we get nothing; yet, if one claim that $a, b, c$ are polynomials?

Theorem 2.1. (Kubota 1972)

This polynomials (in reals) $a(t), b(t)$ and $c(t)$, with max $($ dega, degb) $=$ degc $>0$ define a Pythagorean triple if and only if there exist other three polynomials $u(t), v(t)$ and $w(t)$ such that:

$$
\begin{aligned}
& a(t)=w(t)\left(u^{2}(t) v^{2}(t)\right) \\
& b(t)=2 w(t) u(t) v(t) \\
& c(t)=w(t)\left(u^{2}(t)+v^{2}(t)\right)
\end{aligned}
$$

In what follows, we will suppose that $u$ and $v$ are relatively prime, otherwise the gcd ( greatest common divisor) will be included in $w$. Moreover, suppose that the leading coefficient of $w$ is 1 . Subsequently, we have

$$
\begin{aligned}
& x^{\prime}(t)=w(t)\left(u^{2}(t)-v^{2}(t)\right) \\
& y^{\prime}(t)=2 w(t) u(t) v(t)
\end{aligned}
$$


Some special situations:

a) $w=0$ or $u=v=0$ for any $\mathrm{t} \Rightarrow r$ is a point, not a curve;

b) $u, v, w$ are all constant, $w \neq 0$ (i.e. $w=1$ ) and at least one of $u$ and $v$ is different from $0 \Rightarrow$ on gets a line, uniformly parametrized

ex.:

$$
\begin{aligned}
& x=2 t+3 \\
& y=t-1
\end{aligned}
$$

c) $u, v$ constants, at least one $\neq 0, w(t)$ non constant $\Rightarrow$ one gets a line with non uniform parametric flow

ex.:

$$
\begin{aligned}
& x=t^{3}+1 \\
& y=2 t^{3}-1
\end{aligned}
$$

d) $w(t) \neq 0$ and either $u(t)= \pm v(t) \forall t$ or one of $u$ and $v$ vanishes $\Rightarrow x=0$ (y-axis) or $y=0$ (x-axis)

From now on we suppose $u, v, w \neq 0$ and $\operatorname{gcd}(u, v)=1$ and $u, v$ cannot be simultaneously constant. Denote by $n=\max (\operatorname{deg} x, \operatorname{deg} y) \geq 3$ (the degree of the curve $r) \longrightarrow$ this is a consequence of the hypothesis.

Lemma 4. The degree of a PH curve, given by 2.2, is equal to $n=\lambda+2 \mu+1$, where $\lambda=\operatorname{deg}(w)$, $\mu=\max (\operatorname{deg} u, \operatorname{deg} v)$

Proof. By integration we obtain

$$
\begin{aligned}
& \operatorname{deg}(x) \leq \operatorname{deg} w+2 \max (\operatorname{deg} u, \operatorname{deg} v)+1 \\
& \operatorname{deg}(y)=\operatorname{deg} w+\operatorname{deg} u+\operatorname{deg} v+1
\end{aligned}
$$

Let us note that in $\operatorname{deg}(x)$ we have equality sign if and only if the leading terms do not cancel (computing the expression $u^{2}-v^{2}$ ).

If $\operatorname{deg} u \neq \operatorname{deg} v$ this fact happens and hence $\operatorname{deg} x=\lambda+2 \mu+1$ while $\operatorname{deg} y<\lambda+2 \mu+1$.

If degu $=\operatorname{deg} v=\mu$, then degy $=\lambda+2 \mu+1 \geq \operatorname{deg} x$

Lemma 5. A PH curve of degree $n$ has $n+3$ degree of freedom, while a general polynomial curve of degree $n$ has $2(n+1)$ degrees of freedom.

Proof. Let $\mu=\max (\operatorname{degu}, \operatorname{deg} v) \geq 1$ and $\lambda=\operatorname{deg}(w)$.

Hence, the two polynomials $\mathrm{u}$ and $\mathrm{v}$ have each at must $\mathrm{p}+1$ coefficients. Concerning $w$, one has $\lambda$ coefficients as the leading one is 1 . We conclude that we need $\lambda+2(\mu+1)=n+1$ coefficients to define $x$ and $y$. More, one needs other two integrating constants, meaning other 2 dgrees of freedom. Finally, we have $\mathrm{n}+3$ degrees of freedom.

Nevertheless, these dgrees of freedom are not all available to define the shape of the curve:

* 3 are used for the coordinate system in the plane 
* 2 are used for the parameter; this because the curve $r(\tau)$, where $\tau=p t+q$ ( as change of the parameter) has precisely the same property.

One obtain n-2 for PH curve, comparing with 2n-2 for general polynomial curve.

\section{$2.2 \quad$ PH cubics}

The most simple non trivial PH curves are cubics $(\mathrm{n}=3)$.

We immediately find

$$
\begin{aligned}
& \lambda=\operatorname{deg}(w)=0 \\
& \mu=\max (\operatorname{deg} u, \operatorname{deg} v)=1
\end{aligned}
$$

These curves have only one degree of freedom in shape, comparing with 3 for an arbitrary cubic. Let as study in details these curves, especially their relation with their Bernstein-Bezier form. Let $u$ and $v$ be polynomials of degree 1 given in the following form:

$$
\begin{aligned}
& u(t)=u_{0} b_{0}^{1}(t)+u_{1} b_{1}^{1}(t) \\
& v(t)=v_{0} b_{0}^{1}(t)+v_{1} b_{1}^{1}(t)
\end{aligned}
$$

Moreover $w(t)=1$ Suppose that $\frac{u_{0}}{v_{0}}$ and $\frac{u_{1}}{v_{1}}$ are different.

Compute: $u^{2}(t)-v^{2}(t)=\left(u_{0}^{2}-v_{0}^{2}\right) b_{0}^{2}(t)+\left(u_{0} u_{1}-v_{0} v_{1}\right) b_{1}^{2}(t)+\left(u_{1}^{2}-v_{1}^{2}\right) b_{2}^{2}(t) \rightarrow x^{\prime}(t)$

$2 u(t) v(t)=2 u_{0} v_{0} b_{0}^{2}(t)+\left(u_{0} v_{1}+u_{1} v_{0}\right) b_{1}^{2}(t)+2 u_{1} v_{1} b_{2}^{2}(t) \rightarrow y^{\prime}(t)$

We have $\int_{0}^{t} b_{k}^{n-1}(t) d t=\frac{1}{n} \sum_{j=k+1} n b_{j}^{n}(t), \quad k=0,1, \ldots, n-1$

and hence

$x(t)-x_{0}=\int_{0}^{t}\left(u^{2}-v^{2}\right) d t=\sum_{k=1} 3 x_{k} b_{k}^{3}(t)$

$y(t)-y_{0}=\int_{0}^{t} 2 u(t) v(t) d t=\sum_{k=1} 3 y_{k} b_{k}^{3}(t)$

If we put $P_{0}=\left(x_{0}, y_{0}\right)$ arbitrary point, corresponding to integration constants:

$P_{1}=\left(x_{1}, y_{1}\right), P_{2}=\left(x_{2}, y_{2}\right), P_{3}=\left(x_{3}, y_{3}\right)$ we have

$P_{1}=P_{0}+\frac{1}{3}\left(u_{0}^{2}-v_{0}^{2}, 2 u_{0} v_{0}\right)$

$P_{2}=P_{1}+\frac{1}{3}\left(u_{0} u_{1}-v_{0} v_{1}, u_{0} v_{1}+u_{1} v_{0}\right)$

$P_{3}=P_{2}+\frac{1}{3}\left(u_{1}^{2}-v_{1}^{2}, 2 u_{1} v_{1}\right)$

\section{DO COMPUTATIONS!}

Theorem 2.2. For a planar cubic $r(t)$ given by the Bézier control points $P_{0}, P_{1}, P_{2}, P_{3}$ let $L_{1}, L_{2}, L_{3}$ be the lengths of legs of the Bézier polygon (i.e. $L_{1}=P_{0} P_{1} ; L_{2}=P_{1} P_{2} ; L_{3}=P_{2} P 3$ ) and let $\theta_{1}$ and $\theta_{2}$ be the interior angles in $p_{1}$ and $p_{2}$ in the control polygon. Then the following two conditions are necessary and sufficient $(r(t)$ to be a PH curve:)

a) $L_{2}=\sqrt{L_{1} L_{3}}$

b) $\theta_{1}=\theta_{2}$. 
Proof. Necessity:

$$
\begin{gathered}
w_{0}=u_{0}+i v_{0}, w_{1}=u_{1}+i v_{1} ; \\
w_{0}^{2}=\left(u_{0}^{2}-v_{0}^{2}\right)+2 i u_{0} v_{0} ; \\
w_{0} w_{1}=\left(u_{0} u_{1}-v_{0} v_{1}\right)+i\left(u_{0} v_{1}+u_{1} v_{0}\right) ; \\
w_{1}^{2}=\left(u_{1}^{2}-v_{1}^{2}\right)+2 i u_{1} v_{1} .
\end{gathered}
$$

We obtain:

$$
3 \overrightarrow{P_{0} P_{1}}=w_{0}^{2}, 3 \overrightarrow{P_{1} P_{2}}=w_{0} w_{1}, 3 \overrightarrow{P_{2} P_{3}}=w_{1}^{2} .
$$

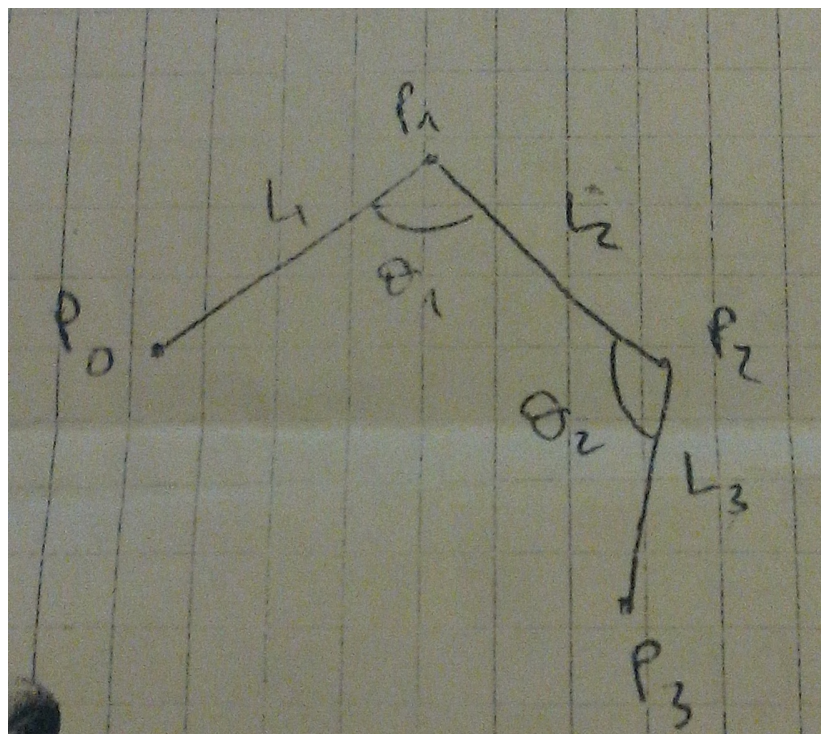

Hence

$$
\begin{gathered}
3 L_{1}=\left|w_{0}\right|^{2}, 3 L_{2}=\left|w_{0}\right|\left|w_{1}\right|, 3 L_{3}=\left|w_{1}\right|^{2} \Rightarrow L_{2}^{2}=L_{1} L_{3} . \\
\cos \left(\theta_{1}\right)=\frac{<\overrightarrow{P_{1} P_{0}}, \overrightarrow{P_{1} P_{2}}>}{\left|\overrightarrow{P_{1} P_{0}}\right|\left|\overrightarrow{P_{1} P_{2}}\right|}=-\frac{1}{9} \frac{<w_{0}^{2}, w_{0} w_{1}>}{L_{1} L_{2}}=-\frac{1}{9 L_{1} L_{2}} \operatorname{Re}\left(w_{0}^{2} \overline{w_{0} w_{1}}\right) \\
=-\frac{\left|w_{0}\right|^{2}}{9 L_{1} L_{2}} \operatorname{Re}\left(w_{0} \overline{w_{1}}\right)=-\frac{\operatorname{Re}\left(w_{0} \overline{w_{1}}\right)}{\left|w_{0}\right|\left|w_{1}\right|}
\end{gathered}
$$

Analogously

$$
\begin{aligned}
& \cos \left(\theta_{2}\right)=-\frac{\operatorname{Re}\left(w_{1} \overline{w_{0}}\right)}{\left|w_{0}\right|\left|w_{1}\right|} \\
& \Rightarrow \cos \left(\theta_{1}\right)=\cos \left(\theta_{2}\right) .
\end{aligned}
$$

$z, w \in \mathbb{C},\langle z, w>=\operatorname{Re}(z, \bar{w}) ;$

$$
\begin{aligned}
& z \times w=\left|\begin{array}{ccc}
x_{1} & x_{2} & i \\
y_{1} & y_{2} & j \\
0 & 0 & k
\end{array}\right|=\left(x_{1} y_{2}-x_{2} y_{1}\right) \vec{k}=-\operatorname{Im}(z \bar{w}) \vec{k} \\
& z \times w=\left|\begin{array}{ccc}
a & 0 & i \\
0 & b & j \\
0 & 0 & k
\end{array}\right|=(a b) \vec{k}=|z||w| \sin \left(\theta_{1}\right) \vec{k}, \theta_{1}=\frac{\pi}{2}
\end{aligned}
$$




$$
\begin{gathered}
W \times w=\left|\begin{array}{ccc}
0 & -a & i \\
b & 0 & j \\
0 & 0 & k
\end{array}\right|=(a b) \vec{k}=|z||w| \sin \left(\theta_{2}\right) \vec{k}, \theta_{2}=\frac{\pi}{2} \\
\sin \left(\theta_{1}\right)=\frac{<\overrightarrow{P_{0} P_{1}} \times \overrightarrow{P_{1} P_{2}}, \vec{k}>}{L_{1} L_{2}}=-\frac{<w_{0}^{2} \times w_{0} w_{1}, \vec{k}>}{9 L_{1} L_{2}} \\
=-\frac{\left|w_{0}^{2}\right|}{9 L_{1} L_{2}}\left(-\operatorname{Im}\left(w_{0} \overline{w_{1}}\right)\right)=\frac{1}{\left|w_{0}\right|\left|w_{1}\right|} \operatorname{Im}\left(w_{0} \overline{w_{1}}\right) \\
\sin \left(\theta_{2}\right)=\frac{<\overline{P_{2} P_{1}} \times \overrightarrow{P_{2} P_{3}}, \vec{k}>}{L_{2} L_{3}}=-\frac{<w_{0} w_{1} \times w_{1}^{2}, \vec{k}>}{9 L_{2} L_{3}} \\
=-\frac{\left|w_{1}^{2}\right|}{9 L_{2} L_{3}}\left(\operatorname{Im}\left(w_{0} \overline{w_{1}}\right)\right)=\frac{1}{\left|w_{0}\right|\left|w_{1}\right|} \operatorname{Im}\left(w_{0} \overline{w_{1}}\right)
\end{gathered}
$$

Theconverse : Let $r(t)$ be a Bézier cubic, given by the control points $p_{0}, \ldots, p_{3}$ and let $L_{1}, L_{2}, L_{3}, \theta_{1}$ and $\theta_{2}$ as before such that (1) $L_{2}^{2}=L_{1} L_{3}$ and (2) $\theta_{1}=\theta_{2}=\theta$ We need to prove that $r$ is a PH curve.

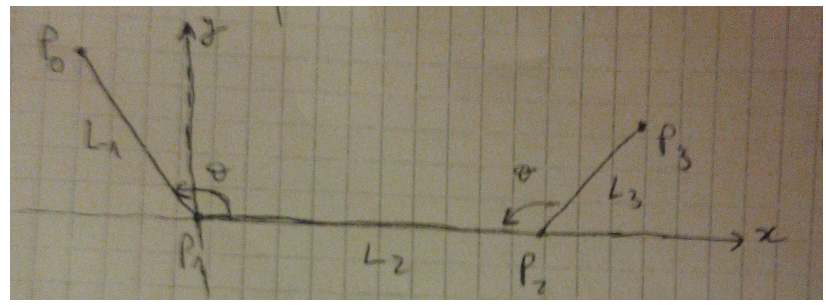

$$
k C_{n}^{k}=n C_{n-1}^{k-1},(n-k) C_{n}^{k}=n C_{n-1}^{k}
$$

Consider the Bézier cubic:

$$
r(t)=(x(t), y(t))=\sum_{k=0}^{3} p_{k} b_{k}^{3}(t) .
$$

Its hodograph may be computed as

$$
\begin{gathered}
\left(x^{\prime}(t), y^{\prime}(t)\right)=\left[\sum_{k=0}^{3} C_{3}^{k} p_{k} t^{k}(1-t)^{3-k}\right]^{\prime} \\
=3 \sum_{k=1}^{3} p_{k} C_{2}^{k-1} p_{k} C_{3}^{k-1} k t^{k-1}(1-t)^{3-k}-\sum_{k=0}^{2} p_{k} C_{3}^{k}(3-k) t^{k}(1-t)^{2-k}= \\
=3 \sum_{k=0}^{2}\left(p_{k+1}-p_{k}\right) b_{k}^{2} .
\end{gathered}
$$

As before, we use complex numbers:

$$
\begin{aligned}
z_{0} & =3 \overrightarrow{p_{0} p_{1}} \\
z_{1} & =3 \overrightarrow{p_{1} p_{2}} \\
z_{2} & =3 \overrightarrow{p_{2} p_{3}} .
\end{aligned}
$$

We write:

$$
\left(x^{\prime}(t), y^{\prime}(t)\right)=\left[\sum_{k=0}^{2} z_{k} b_{k}^{2}(t)\right] .
$$


Legs: $3 L_{1}=\left|z_{0}\right|, 3 L_{2}=\left|z_{1}\right|, 3 L_{3}=\left|z_{2}\right| \Rightarrow^{(1)}\left|z_{1}^{2}\right|=\left|z_{0}\right|\left|z_{2}\right|$. Choose the frame as in the picture above, that is the origin in $P_{1}$, the x-axis in the direction of the vector $\overrightarrow{P_{1} P_{2}}$ :

$$
\begin{gathered}
\overrightarrow{P_{1} P_{0}}=\left(L_{1} \cos \theta, L_{2} \sin \theta\right)=-\frac{1}{3} z_{0} ; \\
\overrightarrow{P_{1} P_{2}}=\left(L_{2}, 0\right)=\frac{1}{3} z_{1} ; \\
\overrightarrow{P_{2} P_{3}}=\left(L_{3} \cos (\pi-\theta), L_{3} \sin (\pi-\theta)\right)=\left(-L_{3} \cos \theta, L_{3} \sin \theta\right)=\frac{1}{3} z_{2} .
\end{gathered}
$$

We get:

$$
\begin{gathered}
x^{\prime}(t)=3\left(-L_{1} \cos \theta b_{0}^{2}+L_{2} b_{1}^{2}-L_{3} \cos \theta b_{2}^{2}\right), \\
y^{\prime}(t)=3\left(-L_{1} \sin \theta b_{0}^{2}+L_{3} \sin \theta b_{2}^{2}\right) .
\end{gathered}
$$

Notice that:

$$
\begin{gathered}
b_{0}^{2}(t)^{2}=b_{0}^{4}(t), b_{1}^{2}(t)=\frac{2}{3} b_{2}^{4}(t), b_{2}^{2}(t)^{2}=b_{4}^{4}(t) \\
b_{0}^{2} b_{2}^{2}=\frac{1}{6} b_{2}^{4}, b_{0}^{2} b_{1}^{2}=\frac{1}{2} b_{1}^{4}, b_{1}^{2} b_{2}^{2}=\frac{1}{2} b_{3}^{4} \\
\frac{1}{9}\left(x^{\prime}(t)+y^{\prime}(t)\right)=\left[L_{1}^{2} \cos ^{2} \theta+L_{1}^{2} \sin ^{2} \theta\right] b_{0}^{2}(t)^{2}+L_{2}^{2} b_{1}^{2}(t)^{2}+\left[L_{3}^{2} \cos ^{2} \theta+L_{3}^{2} \sin ^{2} \theta\right] b_{2}^{2}(t)^{2} \\
-2 L_{1} L_{2} \cos \theta b_{0}^{2} b_{1}^{2}-2 L_{1} L_{3} \cos \theta b_{1}^{2} b_{2}^{2}+-2\left(L_{1} L_{2} \cos ^{2} \theta-L_{1} L_{3} \sin ^{2} \theta\right) b_{0}^{2} b_{2}^{2}= \\
L_{1}^{2} b_{0}^{4}(t)+\frac{2}{3} L_{2}^{2} b_{2}^{4}(t)+L_{3}^{2} b_{4}^{4}(t)-L_{1} L_{2} \cos \theta b_{1}^{4}-L_{2} L_{3} \cos \theta b_{3}^{4}+\frac{1}{3} L_{1} L_{3} \cos 2 \theta b_{2}^{4}=Q(t)^{2},
\end{gathered}
$$

Q-a second order polynomial, $Q=A b_{0}^{2}(t)+B b_{1}^{2}(t)+C b_{2}^{2}(t), A, B, C \in \mathbb{R}$.

$$
Q(t)^{2}=A^{2} b_{0}^{4}(t)+B C b_{3}^{4}(t)+\frac{1}{3} A C b_{2}^{4}(t)
$$

Since $b_{0}^{4}, b_{1}^{4}, b_{2}^{4}, b_{3}^{4}, b_{4}^{4}$ form a basis in $P_{4}$ we should have:

$$
\begin{gathered}
b_{0}^{4}: A^{2}=L_{1}^{2} \Rightarrow A=\varepsilon L_{1}, \varepsilon= \pm 1 ; \\
b_{1}^{4}: A B=-L_{1} L_{2} \cos \theta \Rightarrow B=-\varepsilon L_{2} \cos \theta ; \\
b_{4}^{4}: C^{2}=L_{3}^{2} ; \\
b_{3}^{4}: A C=-L_{2} L_{3} \cos \theta \Rightarrow C=\varepsilon L_{3}
\end{gathered}
$$

(thus the before eq is satisfied);

$$
b_{2}^{4}: \frac{2}{3} B^{2}+\frac{1}{3} A C=\frac{2}{3} L_{2}^{2}+\frac{1}{3} L_{1} L_{3} \cos 2 \theta \Rightarrow 2 L_{2}^{2} \cos ^{2} \theta+L_{1} L_{3}=2 L_{1}^{2}+L_{1} L_{3} \cos 2 \theta
$$

(check this)

$$
2 \cos ^{2} \theta+1=2+\cos 2 \theta(O K)
$$

Hence

$$
\sigma=3\left(L_{1} b_{0}^{2}(t)-L_{2} \cos \theta b_{1}^{2}(t)+L_{3} b_{2}^{2}(t)\right) .
$$




\subsection{The Bernstein Basis}

\section{Definition 2.3.}

$$
\left\{b_{k}^{n}(t)=C_{n}^{k} t^{k}(1-t)^{n-k}, k=\overline{0, n}\right\}
$$

are linearly independent polynomiales.

- We may also generate the Bernstein basis by a simple recursion. Starting with $b_{0}^{0}(t)=1$ one obtains the basis of degree $r+1$ from that of degree $r$

$$
b_{k}^{r+1}=t b_{k-1}^{r}(t)+(1-t) b_{k}^{r}(t), k=\overline{0, r+1}
$$

with the convention $b_{k}^{r}(t) \equiv 0$ if $k<0$ or if $k>r$.

\section{$\underline{E x}:$}

$-\underline{r}=0$

$$
\begin{gathered}
b_{0}^{1}(t)=t b_{-1}^{0}(t)+(1-t) b_{0}^{0}(t)=1-t \\
b_{1}^{1}(t)=t b_{0}^{0}(t)+(1-t) b_{1}^{0}(t)=t
\end{gathered}
$$

$-\underline{r=1}$

$$
\begin{gathered}
b_{0}^{2}(t)=t b_{-1}^{1}(t)+(1-t) b_{0}^{1}(t)=(1-t)^{2} \\
b_{1}^{2}(t)=t b_{0}^{1}(t)+(1-t) b_{1}^{1}(t)=2 t(1-t) \\
b_{1}^{2}(t)=t b_{2}^{1}(t)+(1-t) b_{2}^{1}(t)=t^{2}
\end{gathered}
$$

- Derivatives and integrals

$$
\begin{aligned}
& \frac{d}{d t} b_{k}^{n}(t)=n\left[b_{k-1}^{n-1}(t)-b_{k}^{n-1}(t)\right], k=\overline{0, n} \\
& \int b_{k}^{n}(t) d t=\frac{1}{n+1} \sum_{j=k+1}^{n+1} b_{j}^{n+1}(t), k=\overline{0, n}
\end{aligned}
$$

- Let $p_{0}, p_{1}, \ldots, p_{n}$ be a set of $n+1$ points in the plane $\rightarrow$ control points defining the control polygon. Consider

$$
b(t)=\sum_{k=0}^{n} p_{k} b_{k}^{n}(t)
$$

It is called the Bézier curve of degree $\mathrm{n}$.

Lemma 6. A straight line may never intersect a Bézier curve more ofen then it intersects the control polygon of that curve.

- de Casteljau algorithm

- Bézier curve hodographs:

$$
h(t)=\sum_{k=0}^{n-1} n\left(p_{k+1}-p_{k}\right) b_{k}^{n-1}(t) .
$$




\subsection{Spatial $\mathrm{PH}$ curves}

Let

$$
\left\{\begin{array}{l}
r: I \rightarrow \mathbb{R}^{3} \\
r=r(t)=(x(t), y(t))
\end{array} ;\right.
$$

a polynomial curve and $r^{\prime}$ its hodograph. The condition for $r$ to be a PH curve writes as

$$
(P H): x^{\prime}(t)^{2}+y^{\prime}(t)^{2}+z^{\prime}(t)^{2}=\sigma(t)^{2}
$$

for any $t \in I$ for a certain polynomial $\sigma$. A sufficient condition for $r$ to satisfy $(P H)$ is that coordinate functions $x, y$, and $z$ satisfy:

$$
*\left\{\begin{array}{l}
x^{\prime}(t)=2 h(t) u(t) v(t) \\
y^{\prime}(t)=2 h(t) u(t) w(t) \\
z^{\prime}(t)=h(t)\left[u^{2}(t)-v^{2}(t)-w^{2}(t)\right]
\end{array} ;\right.
$$

It follows then

$$
\sigma(t)=h(t)\left[u^{2}(t)+v^{2}(t)+w^{2}(t)\right]
$$

Similar conditions, as in the planar case, may be asked:

(a) h, u, v, w do not vanish simultaneously

(b) u, v, w are not all constant

(c) $g c b(u(t), v(t), w(t))=1($ constant, not a polynomial)

Yet, the condition $(*)$ is not necessary; in fact, does not exist an easy necessary and sufficient condition for spatial $\mathrm{PH}$ curves.

To justify the previous statement, let us consider the following hodograph:

$$
\begin{gathered}
x^{\prime}=(1-t)^{2} \\
y^{\prime}=t^{2} \\
z^{\prime}=1
\end{gathered}
$$

which is Pythagorean since there exists

$$
\sigma(t)=\sqrt{2}\left(t^{2}-t+1\right)
$$

Prove this!

Let's see that $(*)$ cannot be satisfied. We prove this by contradiction. Obviously, $h=1$. Therefore, we are looking for $u, v, w$ (three polynomials) s.t.

$$
\left\{\begin{array}{l}
2 u v=(1-t)^{2} \\
2 u w=t^{2} \\
u^{2}-v^{2}=1
\end{array} ;\right.
$$

First we have

$$
\begin{gathered}
\left\{\begin{array}{l}
4 u^{2} v^{2}=(1-t)^{4} \\
4^{2} w^{2}=t^{4}
\end{array}\right. \\
4 u^{2}\left(v^{2}+w^{2}\right)=t^{4}+(1-t)^{4}
\end{gathered}
$$


Then, we write

$$
v^{2}+w^{2}=u^{2}-1
$$

We obtain

$$
4 u^{2}\left(u^{2}-1\right)=t^{4}+(1-t)^{4} .
$$

It follows that, if $\mathrm{n}$ exists, it should be a polynomial of degree 1 , that is $u(t)=a(t)+b$. For

$$
\begin{gathered}
t=0: 4 b^{2}\left(b^{2}-1\right)=1 \rightarrow b^{2}=\frac{1+\sqrt{2}}{2} \\
t=1: 4 u(1)^{2}\left(u^{2}(1)-1\right)=1 \rightarrow u^{2}(1)=\frac{1+\sqrt{2}}{2} \\
t=\frac{1}{2} \rightarrow 4 u^{2}\left(\frac{1}{2}\right)\left(u^{2}\left(\frac{1}{2}\right)-1\right)=\frac{1}{8} \\
u\left(\frac{1}{2}\right)=\frac{a}{2}+b=\frac{a+2 b}{2}=0 \rightarrow a=-2 b
\end{gathered}
$$

(these are contradictory) Moreover, we have to prove also that $u, v, w$ do not exist for any other permutation of $x, y$ and $z$. For example:

- $x \leftrightarrow y$ yields nothing important

- $x \leftrightarrow z$ yields

$$
\left\{\begin{array}{l}
2 u v=1 \\
2 u w=t^{2} \\
u^{2}-v^{2}-w^{2}=(1-t)^{2}
\end{array} ;\right.
$$

One obtains $\mathrm{u}, \mathrm{v}$ are constants:

$$
u=\frac{c}{2}, v=\frac{1}{c}, c \neq 0 .
$$

Then, $w=\frac{t^{2}}{c}$. Finally $\frac{c^{2}}{4}-\frac{1}{c^{2}}-\frac{t^{4}}{c^{2}}=(1-t)^{2}$. 


\subsection{Spatial PH cubics}

We give a geometric characterization of spatial $\mathrm{PH}$ cubics in terms of the geometry of the corresponding Bézier polygon.

Let $p_{0}, p_{1}, p_{2}$ and $p_{3}$ be the control points defining the cubic. Denote by $\overrightarrow{L_{k}}=p_{k}-p_{k-1}, k=$ $1,2,3$, i.e. $\overrightarrow{L_{k}}=\overrightarrow{p_{k-1} p_{k}}$ and $L_{k}$ the length of the vector $\overrightarrow{L_{k}}$.

Theorem 2.3. A spatial cubic defined by the Bézier control points $p_{0}, p_{1}, p_{2}$ and $p_{3}$ is a PH cubic if and only $\overrightarrow{L_{1}}$ and $\overrightarrow{L_{3}}$ lie on a right circular cone having $\overrightarrow{L_{2}}$ as axis, and they azimuthal separation $\varphi$, on the cone, is given by

$$
\cos \varphi=1-\frac{2 L_{2}}{L_{1} L_{3}}
$$

Proof. $\Leftarrow$ Suppose that the control points are given as in the theorem. Choose the coordinate system s.t. OZ be determined by $p_{1}$ and $p_{2}$, more precisely $p_{1}=(0,0,0)$ and $p_{2}=\left(0,0, L_{2}\right)$ (We have already done a translation and a rotation). Now, one can rotate the Bézier control polygon around $\mathrm{OZ}$ axis until $p_{0}$ belongs to the plane $x \mathrm{Oz}$. Denote by $2 \theta$ the angle of the cone:

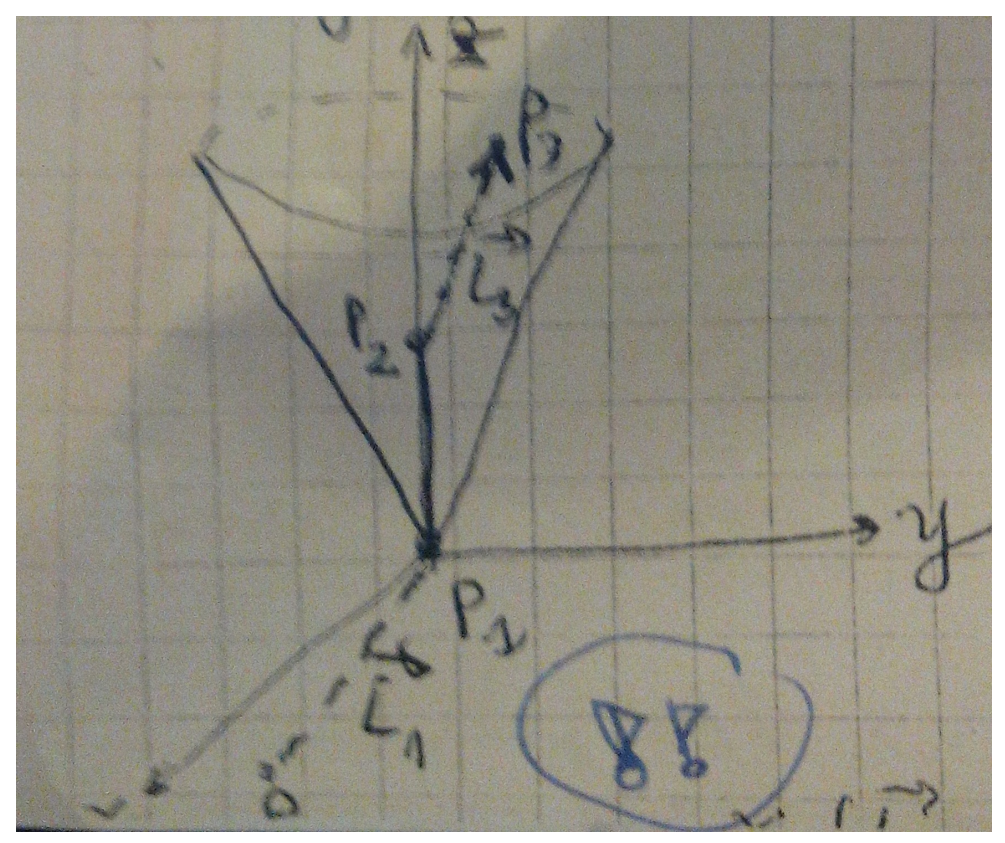

EQ of the cone:

$$
\begin{gathered}
x^{2}+y^{2}=z^{2} \tan ^{2} \theta \\
p_{0} \in \text { cone } \cap O z \Rightarrow x_{0}^{2}=z_{0}^{2} \tan ^{2} \theta \\
\left|\overrightarrow{P_{0} P_{1}}\right|=L_{1} \Rightarrow x^{2}+y_{0}^{2}+z_{0}^{2}=L_{1}^{2} \Rightarrow z_{0}^{2}=L_{1}^{2} \cos ^{2} \theta \Rightarrow z_{0}=\varepsilon L_{1} \cos \theta, \varepsilon= \pm 1 \\
\prec\left(\overrightarrow{L_{1}}, O Z\right)=\theta \Rightarrow \varepsilon=-1 \\
x_{0}=\bar{\varepsilon} L_{1} \sin \theta, \bar{\varepsilon}= \pm 1 . \\
P_{0}=\left(\bar{\varepsilon} L, \sin \theta, 0, L_{1} \cos \theta\right) \\
P_{1}=(0,0,0) \\
P_{2}=\left(0,0, L_{2}\right)
\end{gathered}
$$




$$
\overrightarrow{L_{2}}=L_{2}(0,0,1)
$$

Concerning $P_{3}$, it is a point in the space, such that the vector $\overrightarrow{L_{3}}$, applied at the origin, is a vector on the cone. Hence, the angle $\prec\left(O Z, \overrightarrow{L_{3}}\right)=\theta$. Hence, the last component of $\overrightarrow{L_{3}}$ is $L_{3} \cos \theta$.

$$
\begin{gathered}
\overrightarrow{L_{1}}=\left(-3 \bar{\varepsilon} L_{1} \sin \theta, 0, L_{1} \cos \theta\right)=L_{1}(-\bar{\varepsilon} \sin \theta, 0, \cos \theta) \\
\overrightarrow{L_{3}}=L_{3}(u, v, \cos \theta) \text { withu }{ }^{2}+v^{2}=\sin ^{2} \theta
\end{gathered}
$$

$\varphi$ is the angle between the projections of the two vectors on $x O y$ ( $\perp$ on the cone axis).

$$
\begin{gathered}
\operatorname{pr}\left(\overrightarrow{L_{1}}=L_{1}(-2 \sin \theta, 0,0)\right. \\
\operatorname{pr}\left(\overrightarrow{L_{3}}=L_{3}(u, v, 0)\right. \\
\cos \varphi=\frac{<(-\bar{\varepsilon} \sin \theta, 0,0),(u, v, 0)>}{\sin \theta \sqrt{u^{2}+v^{2}}}=\frac{-\bar{\varepsilon} u}{\sin \theta} \Rightarrow u=-\bar{\varepsilon} \sin \theta \\
u^{2}+v^{2}=\sin ^{2} \theta \Rightarrow v= \pm \bar{\varepsilon} \sin \theta \sin \varphi, \pm \varepsilon=\epsilon \\
\overrightarrow{L_{3}}=L_{3}\left(-3 \bar{\varepsilon} L_{1} \sin \theta \cos \varphi, \epsilon \sin \theta \sin \varphi, \cos \theta\right)
\end{gathered}
$$

As in the planar case

$$
\begin{gathered}
r^{\prime}(t)=3\left[(1-t)^{2} \overrightarrow{L_{1}}+2 t(1-t) \overrightarrow{L_{2}}+t^{2} \overrightarrow{L_{3}}\right] \\
x^{\prime}=-3 \varepsilon\left[(1-t)^{2} L_{1} \sin \theta+t^{2} L_{3} \sin \theta \cos \varphi\right. \\
y^{\prime}=3 \epsilon 2 t(1-t) L_{3} \sin \theta \sin \varphi \\
z^{\prime}=3\left[\left(1-t^{2}\right) L_{1} \cos \theta+2 t(1-t) L_{2}+t^{2} L_{3} \cos \theta\right]
\end{gathered}
$$

